TRANSACTIONS OF THE

AMERICAN MATHEMATICAL SOCIETY

Volume 350, Number 12, December 1998, Pages 4775-4798

S $0002-9947(98) 02403-9$

\title{
THE STABLE HOMOTOPY TYPES OF STUNTED LENS SPACES MOD 4
}

\author{
HUAJIAN YANG
}

\begin{abstract}
Let $L_{n}^{n+k}$ be the mod 4 stunted lens space $L^{n+k} / L^{n-1}$. Let $\nu(m)$ denote the exponent of 2 in $m$, and $\phi(k)$ the number of integers $j$ satisfying $j \equiv$ $0,1,2,4(\bmod 8)$, and $0<j \leq k$. In this paper we complete the classification of the stable homotopy types of mod 4 stunted lens spaces. The main result (Theorem 1.3 (i)) is that, under some appropriate conditions, $L_{n}^{n+k}$ and $L_{m}^{m+k}$ are stably equivalent iff $\nu(n-m) \geq \phi(k)+\delta$, where $\delta=-1,0$ or 1 .
\end{abstract}

\section{INTRODUCTION}

Let $L_{n}^{n+k}=L^{n+k} / L^{n-1}$ and $P_{n}^{n+k}=P^{n+k} / P^{n-1}$ be respectively the mod 4 stunted lens space and the stunted real projective space. Let $\nu(m)$ denote the exponent of 2 in $m$, and $\phi(k)$ the number of integers $j$ satisfying $j \equiv 0,1,2,4(\bmod 8)$, and $0<j \leq k$. Two stunted lens spaces $L_{n}^{n+k}$ and $L_{m}^{m+k}$ are said to be stably equivalent, denoted by $L_{n}^{n+k} \sim L_{m}^{m+k}$, if there exists a homotopy equivalence $\Sigma^{N} L_{n}^{n+k} \rightarrow \Sigma^{N+n-m} L_{m}^{m+k}$ for some $N$.

Classification of the stable homotopy types of stunted real projective spaces was begun by Feder, Gitler, and Mahowald in 1977 ([7]), and finished by Davis and Mahowald in 1986 ([4]). For an odd prime $p$, the classification of $\bmod p$ stunted lens spaces was recently finished by Gonzalez $([9])$. The classification of mod $2^{r}$ stunted lens spaces, in particular the mod 4 stunted lens spaces, has been a favorite subject of some mathematicians for many years ([8], [11], [13], [14], [20]).

Let $\delta(n, k)$ and $\epsilon(n, k)$ be functions defined by the $\bmod 4$ values of $n$ and the mod 8 values of $k$, as given by the following tables:

\begin{tabular}{c|cccc}
$k \equiv$ & 4 & 5 & 6 & 7 \\
\hline 0 & 3 & 3 & 3 & 3 \\
1 & 3 & 3 & 3 & 4 \\
2 & 3 & 3 & 4 & 4 \\
3 & 3 & 4 & 4 & 4
\end{tabular}

Function $\epsilon(n, k)$

\begin{tabular}{c|cccccccc}
$k \equiv$ & 0 & 1 & 2 & 3 & 4 & 5 & 6 & 7 \\
\hline 0 & 0 & -1 & -1 & -1 & -1 & -1 & 0 & 0 \\
1 & 0 & 0 & -1 & 0 & -1 & 0 & 0 & 1 \\
2 & 0 & -1 & 0 & 0 & -1 & -1 & 1 & 1 \\
3 & 0 & 0 & 0 & 0 & -1 & 1 & 1 & 1
\end{tabular}

Function $\delta(n, k)$

Received by the editors June 6, 1995

1991 Mathematics Subject Classification. Primary 55T15, 55T25. 
Recall that $L_{n}^{n+k}$ is $S$-coreducible if there exists a map $f: \Sigma^{N} L_{n}^{n+k} \rightarrow S^{N+n}$ for some $N \geq 0$ such that the composite $S^{N+n} \stackrel{i}{\rightarrow} \Sigma^{N} L_{n}^{n+k} \stackrel{f}{\rightarrow} S^{N+n}$ is of degree one, where $i$ is the inclusion to the bottom cell. While $L_{n}^{n+k}$ is $S$-reducible if there is a map $g: S^{N+n+k} \rightarrow \Sigma^{N} L_{n}^{n+k}$ for some $N \geq 0$ such that $S^{N+n+k} \stackrel{g}{\rightarrow} \Sigma^{N} L_{n}^{n+k} \stackrel{p}{\rightarrow} S^{N+n+k}$ is of degree one, where $p$ is the standard projection. It is known that the $S$-dual of $L_{n}^{n+k}$ is $\Sigma L_{-n-k-1}^{-n-1}$. Thus it is clear that $L_{n}^{n+k}$ is $S$-coreducible iff its $S$-dual $D L_{n}^{n+k}$ is $S$-reducible.

In this paper we complete the classification of the stable homotopy types of mod 4 stunted lens spaces by giving proofs to the following Theorems 1.1-1.3. Here Theorem 1.1 follows from the order of $J\left(\lambda_{k}-2\right)$ in $J\left(L^{k}\right)$, the $J$-group of $L^{k}$. In Theorem 1.3 , results for $n \equiv 2(\bmod 4)$ and $k \equiv 2,3,6,7(\bmod 8) ; n \equiv 3(\bmod 4)$ and $k \equiv 2,5,6(\bmod 8)$; can be found in $[8$, Theorem 1.6$]$ and $[13$, Theorem 1$]$.

Theorem 1.1. (i) Let $0<k<4$. Then $L_{n}^{n+k} \sim L_{m}^{m+k}$ iff $\nu(n-m) \geq 1$ when $k=1$, and 2 when $k=2,3$.

(ii) Let $k \geq 4$. Then $L_{n}^{n+k}$ is $S$-coreducible (resp. S-reducible) iff

$$
\nu(n)(\text { resp. } \nu(n+k+1)) \geq \begin{cases}\phi(k)+1 & \text { when } k \equiv 0,1,4,5,6,7(\bmod 8), \\ \phi(k) & \text { when } k \equiv 2,3(\bmod 8) .\end{cases}
$$

(iii) Let $k \geq 4$. Suppose either $L_{n}^{n+k}$ or $L_{m}^{m+k}$ is $S$-coreducible (or S-reducible). Then $L_{n}^{n+k} \sim L_{m}^{m+k}$ iff

$$
\nu(n-m) \geq \begin{cases}\phi(k)+1 & \text { when } k \equiv 0,1,4,5,6,7(\bmod 8) \\ \phi(k) & \text { when } k \equiv 2,3(\bmod 8)\end{cases}
$$

Theorem 1.2. Let $4 \leq k<8$. Suppose neither of $L_{n}^{n+k}, L_{m}^{m+k}$ is $S$-coreducible nor $S$-reducible. Then $L_{n}^{n+k} \sim L_{m}^{m+k}$ iff $\nu(n-m) \geq \epsilon(n, k)$.

Theorem 1.3. Let $k \geq 8$. Suppose neither of $L_{n}^{n+k}, L_{m}^{m+k}$ is $S$-coreducible nor $S$-reducible.

(i) If none of $\{\nu(n), \nu(m), \nu(n+k+1), \nu(m+k+1)\}$ is

$$
\begin{cases}4 b+2 & \text { when } k=8 b+4,5 \\ 4 b & \text { when } k=8 b+1\end{cases}
$$

then $L_{n}^{n+k} \sim L_{m}^{m+k}$ iff $\nu(n-m) \geq \phi(k)+\delta(n, k)$.

(ii) If one of $\{\nu(n), \nu(m), \nu(n+k+1), \nu(m+k+1)\}$ is $4 b+2$ when $k=8 b+4,5$, or is $4 b$ when $k=8 b+1$, then $L_{n}^{n+k} \sim L_{m}^{m+k}$ iff $\nu(n-m) \geq 4 b+3$ when $k=8 b+4,5$, or $\nu(n-m) \geq 4 b+1$ when $k=8 b+1$.

The paper is organized as follows. In section 2, we study the Adams operation $\Psi^{3}$ on KO-homology. Theorems 1.1-1.3 are proved in section 3 assuming sections 4-6. Results in section 4 on $J$-homology and coextension will be used in section 6 . In section 6 , we study the triviality of the composite

$$
\tilde{\beta}_{t}: S^{2 n+1+t} \stackrel{a \beta_{t}}{\longrightarrow} S^{2 n+1} \rightarrow S^{2 n+1} \vee L_{2 n-b}^{2 n} \rightarrow L_{2 n-b}^{2 n+t+c},
$$

where $a, c, t$ are positive integers, and $b \geq 0$ is an appropriate integer such that $L_{2 n-b}^{2 n+1}$ is $S$-reducible, while $\beta_{t}$ is the generator of $I m J$ on a $t$-stem. In section 5 , we study the Adams filtration of $\tilde{\beta}_{8 b-1}$. As in [4], the determination of whether the element $\tilde{\beta}_{t}$ is null-homotopic will be crucial. $J$-homology, Adams spectral sequence (ASS), and Mahowald's table 8.1 in [16], will be used actively. 
The paper is substantially the author's Ph.D thesis under Donald M Davis. The author is very grateful to Professor Davis for his constant encouragement and enthusiastic guidance. The author thanks the referee for many valuable suggestions, and Dr. Kono for informing him of his result [13, Theorem 2 (ii)], which leads to our Theorem 1.3 (ii) here.

\section{2. $K O$-HOMOLOGY}

Lemma 2.1. Let $f: L_{n}^{n+k} \rightarrow \Sigma^{n-m} L_{m}^{m+k}$ be a stable equivalence and $\nu(n-m) \geq 3$.

(i) Let $\omega \in K O^{-4 j}\left(\Sigma^{n-m} L_{m}^{m+k}\right)$ be of order $2^{e}$. Suppose $\Psi^{3}(\omega)=3^{2 j+(n-m) / 2} \omega$ and $\Psi^{3}\left(f^{*}(\omega)\right)=3^{2 j} f^{*}(\omega)$. Then $\nu(n-m) \geq e-1$.

(ii) Let $\omega \in K O_{4 j-1}\left(L_{n}^{n+k}\right)$ be of order $2^{e}$. Suppose $\Psi^{3}(\omega)=3^{-2 j} \omega$ and $\Psi^{3} f_{*}(\omega)=3^{-2 j-(n-m) / 2} f_{*}(\omega)$. Then $\nu(n-m) \geq e-1$.

Proof. Notice that (ii) is dual to (i). For (i), we have $\left(3^{-(n-m) / 2}-1\right) \omega=0$ since $\Psi^{3} f^{*}(\omega)=f^{*}\left(\Psi^{3}(\omega)\right)$. Thus $\nu(n-m) \geq e-1$ by [1, Lemma 8.1].

Let $L^{\infty}$ be the mod 4 infinite lens space with a $C W$-structure as indicated in $\left[21\right.$, p. 91]. It is known that $H^{*}\left(L^{\infty} ; \mathbf{Z}_{2}\right) \approx \mathbf{Z}_{2}[u, v] /\left(u^{2}\right)$, where $\operatorname{deg} u=1$ and $\operatorname{deg} v=2$, satisfying

$$
\mathrm{Sq}^{2 i}\left(v^{j}\right)=\left(\begin{array}{l}
j \\
i
\end{array}\right) v^{i+j}, \quad \mathrm{Sq}^{2 i}\left(u v^{j}\right)=\left(\begin{array}{l}
j \\
i
\end{array}\right) u v^{i+j}, \quad \mathrm{Sq}^{2 i+1}(-)=0 .
$$

Let $\rho: P^{\infty} \rightarrow L^{\infty}$ be the covering map. Then $\rho$ induces a stable map $P_{n}^{n+k} \rightarrow L_{n}^{n+k}$ denoted also by $\rho$. The following lemma is immediate.

Lemma 2.2. (i) The map $\rho: P_{m}^{m+k} \rightarrow L_{m}^{m+k}$ is of odd degree on even dimensional cells, and even degree on odd dimensional cells.

(ii) If $m \leq 2 n+1 \leq m+k$, then $\rho_{*}: H_{2 n+1}\left(P_{m}^{m+k}\right.$; $\left.\mathbf{Z}\right) \rightarrow H_{2 n+1}\left(L_{m}^{m+k}\right.$; $\left.\mathbf{Z}\right)$ is a monomorphism $\mathbf{Z}_{2} \rightarrow \mathbf{Z}_{4}$, and $\rho_{*}: H_{2 n+1}\left(P_{m}^{m+k} ; \mathbf{Z}_{2}\right) \rightarrow H_{2 n+1}\left(L_{m}^{m+k} ; \mathbf{Z}_{2}\right)$ is trivial, while $\rho_{*}: H_{2 n}\left(P_{m}^{m+k} ; \mathbf{Z}_{2}\right) \rightarrow H_{2 n}\left(L_{m}^{m+k} ; \mathbf{Z}_{2}\right)$ is an isomorphism.

(iii) If $m \leq 2 n \leq m+k$, then $\rho^{*}: H^{2 n}\left(L_{m}^{m+k} ; \mathbf{Z}\right) \rightarrow H^{2 n}\left(P_{m}^{m+k} ; \mathbf{Z}\right)$ is an epimorphism $\mathbf{Z}_{4} \rightarrow \mathbf{Z}_{2}$, and $\rho^{*}: H^{2 n}\left(L_{m}^{m+k} ; \mathbf{Z}_{2}\right) \rightarrow H^{2 n}\left(P_{m}^{m+k} ; \mathbf{Z}_{2}\right)$ is an isomorphism, while $\rho^{*}: H^{2 n+1}\left(L_{m}^{m+k} ; \mathbf{Z}_{2}\right) \rightarrow H^{2 n+1}\left(P_{m}^{m+k} ; \mathbf{Z}_{2}\right)$ is trivial.

Lemma $2.3([20,2.5])$. Let $\left\{E_{2}^{p, q}, d_{r}\right\}$ be the Atiyah-Hirzebruch spectral sequence (AHSS) for $K O^{*}\left(L_{m}^{n}\right)$ with $E_{2}^{p, q}=H^{p}\left(L_{m}^{n} ; K O^{q}(p t)\right)$. Then differentials $d_{2}^{i, 8 k}$ : $E_{2}^{i, 8 k} \rightarrow E_{2}^{i+2,8 k-1}, \quad d_{2}^{i, 8 k-1}: E_{2}^{i, 8 k-1} \rightarrow E_{2}^{i+2,8 k-2}$ and $d_{3}^{i, 8 k-2}: E_{3}^{i, 8 k-2} \rightarrow E_{3}^{i+3,8 k-4}$ are given by $\mathrm{Sq}^{2} \rho_{2}, \mathrm{Sq}^{2}$ and $\beta_{2} \mathrm{Sq}^{2}$ respectively, where $\rho_{2}$ is the mod 2 reduction, and $\beta_{2}$ is the Bockstein operation associated with the exact sequence $0 \rightarrow \mathbf{Z} \rightarrow \mathbf{Z} \rightarrow$ $\mathbf{Z}_{2} \rightarrow 0$.

Let $\bar{\eta} \in \widetilde{K O}(-)$ (or $\widetilde{K}(-))$ denote the reduction of a vector bundle of $\eta$. Let $\lambda_{k}$ be the realification of the complex Hopf bundle $\xi_{k}$ over $L^{k}$. The next lemma is from [14, Prop. 3.3 (2)] and [8, Theorem 2.5].

Lemma 2.4. (i) The order of $\bar{\xi}_{k}$ in $\widetilde{K}\left(L^{k}\right)$ is $2^{[k / 2]+1}$.

(ii) If $k \geq 4$, then the order of $\left(\bar{\lambda}_{k}\right)^{i}$ in $\widehat{K O}\left(L^{k}\right)$ is

$$
\begin{cases}2^{2+[k / 2]-2 i+1} & \text { if } k \equiv 1(\bmod 4) \text { or } k \equiv 4(\bmod 8), \\ 2^{2+[k / 2]-2 i} & \text { otherwise. }\end{cases}
$$


For a given $k$, let $F^{n}(X)$ be the subgroup of $K O_{k}(X)$ (or $K O^{k}(X)$ ) of elements of $C W$-filtrations $\geq n$ in AHSS.

Lemma 2.5. $\widetilde{K O}\left(L^{2}\right) \approx \widetilde{K O}\left(L^{3}\right) \approx \mathbf{Z}_{2} \oplus \mathbf{Z}_{2}$.

Proof. By AHSS, we see that $\widetilde{K O}\left(L^{2}\right) \approx \widetilde{K O}\left(L^{3}\right)$, and $\widetilde{K O}\left(L^{2}\right)$ is of order 4 . Thus it suffices to show $2 x=0$ for $x \in \widetilde{K O}\left(L^{2}\right)$. The left square

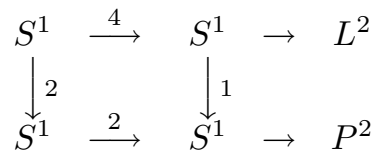

commutes. Thus there exists a stable map $f: L^{2} \rightarrow P^{2}$ whose degrees on the top and bottom cells are respectively 2 and 1 . It is known that $\widehat{K O}\left(P^{2}\right) \approx \mathbf{Z}_{4}$. If $\widetilde{K O}\left(L^{2}\right) \approx \mathbf{Z}_{4}$, then any element of $\widetilde{K O}\left(L^{2}\right)$ from $F^{1}\left(L^{2}\right)$ would be a generator, and $f^{*}: \widetilde{K O}\left(P^{2}\right) \rightarrow \widetilde{K O}\left(L^{2}\right)$ would be an isomorphism because $f$ is of degree 1 on the bottom cell. But it is not since $f$ is of degree 2 on the top cell.

We assume in the following that $L_{m}^{n}$ is the base point if $m>n$. Suppose $p$ is either the composite $L^{k} \rightarrow L_{8 b}^{k}=S^{8 b} \vee L_{8 b+1}^{k} \rightarrow S^{8 b}$ when $8 b \leq k \leq 8 b+3$, or $L^{k} \rightarrow L_{8 b+4}^{k}=S^{8 b+4} \vee L_{8 b+5}^{k} \rightarrow S^{8 b+4}$ when $8 b+4 \leq k \leq 8 b+7$.

Theorem 2.6. Let $g_{1}, g_{2}$ generate $\widetilde{K O}\left(S^{8 b}\right), \widetilde{K O}\left(S^{8 b+4}\right)$ respectively.

(i) If $k=8 b, 8 b+1$, then there exists an odd integer $a_{1}$ and a stable vector bundle $\eta_{1}$ over $S^{8 b}$ such that $\eta_{1}=a_{1} g_{1}$, and $2^{4 b-1} \bar{\lambda}_{k}=p^{*}\left(2 \eta_{1}\right)$. Moreover, $2^{4 b-1} \bar{\lambda}_{k} \in$ $\widetilde{K O}\left(L^{k}\right)$ is of order 2 (resp. 4 ) if $k=8 b$ (resp. $8 b+1$ ).

(ii) If $k=8 b+2,8 b+3$, then there exists an odd integer $a_{2}$ and a stable vector bundle $\eta_{2}$ over $S^{8 b}$ such that $\eta_{2}=a_{2} g_{1}$ in $\widetilde{K O}\left(S^{8 b}\right)$, and $2^{4 b} \bar{\lambda}_{k}=p^{*}\left(4 \eta_{2}\right)$. Moreover, $2^{4 b} \bar{\lambda}_{k} \in \widetilde{K O}\left(L^{k}\right)$ is of order 2 .

(iii) If $8 b+4 \leq k \leq 8 b+7$, then there exists an odd integer $a_{3}$ and a stable vector bundle $\eta_{3}$ over $S^{8 b+4}$ such that $\eta_{3}=a_{3} g_{2}$, and $2^{4 b+1} \bar{\lambda}_{k}=p^{*}\left(\eta_{3}\right)$. Moreover, $2^{4 b+1} \bar{\lambda}_{k} \in \widetilde{K O}\left(L^{k}\right)$ is of order 4 .

Proof. The orders of the claimed elements follows from Lemma 2.4.

Let $\left\{E_{r}^{p, q}(i), d_{r}^{(i)}\right\}, i=1,2$, be respectively the AHSSs for $K^{*}\left(L^{k}\right)$ and $K O^{*}\left(L^{k}\right)$. By [20, p. 240, (1)], elements of $E_{2}^{p, q}(i)$ survive to $E_{\infty}$ if $p+q \equiv 0(\bmod 4)$.

Consider (i). By Lemma 2.4, $2^{4 b-1} \lambda_{k}$ is stably trivial over $L^{8 b-1}$. Thus there is a stable vector bundle $\omega_{1}$ over $L_{8 b}^{k}$ such that $2^{4 b-1} \bar{\lambda}_{k}=p_{1}^{*}\left(\omega_{1}\right)$, where $p_{1}: L^{k} \rightarrow L_{8 b}^{k}$ is the standard projection. In this case $L_{8 b}^{k}=S^{8 b} \vee L_{8 b+1}^{k}$ and $\widetilde{K O}\left(L_{8 b+1}^{k}\right)$ is either trivial or $\mathbf{Z}_{2}$. Since $2^{4 b} \bar{\lambda}_{k}$ is trivial over $L^{8 b}$ and is of order 2 over $L^{8 b+1}$, we see that $\widetilde{K O}\left(S^{8 b+1}\right) \longrightarrow \widetilde{K O}\left(L^{8 b+1}\right)$ maps the generator to $2^{4 b} \bar{\lambda}_{k}$. Thus we can ignore the part $L_{8 b+1}^{k}$ and let $\omega_{2}$ be the restriction of $\omega_{1}$ on $S^{8 b}$. Then $\omega_{2}$ is not divisible by 4 , otherwise $p^{*}\left(\omega_{2}\right)$ is trivial over $L^{8 b}$ and $p^{*}\left(\omega_{1}\right) \in \widetilde{K O}\left(L^{8 b+1}\right)$ is of order $\leq 2$. This is contrary to Lemma 2.4. Next we claim that $\omega_{2} \in \widetilde{K O}\left(S^{8 b}\right)$ is divisible by 2 , which will imply (i). Note that $2^{4 b-1} \bar{\lambda}_{k} \in F^{8 b}$. If $\omega_{2}$ is not divisible by 2 , then $2^{4 b-1} \bar{\lambda}_{k}$ generates $F^{8 b} / F^{8 b+1} \approx \mathbf{Z}_{4}$. This means $2^{4 b} \bar{\lambda}_{k}$ is of order 2 in $F^{8 b} / F^{8 b+1}$. However, we will show that $2^{4 b} \bar{\lambda}_{k}$ is trivial in $F^{8 b} / F^{8 b+1}$.

Recall that $2^{4 b} \lambda_{k}$ is the realification of $2^{4 b} \xi_{k}$, where $\xi_{k}$ is the complex Hopf bundle over $L^{k}$, and $2^{4 b} \xi_{k}$ is stably trivial over $L^{8 b-1}$ by Lemma 2.4 . Since $2^{4 b+1} \xi_{8 b+1}$ is stably trivial over $L^{8 b+1}, 2^{4 b} \bar{\xi}_{k}$ corresponds to an element of $E_{2}^{8 b,-8 b}(1)$ of order 2 . 
By $[19$, p. 304$]$ the realification $\pi_{8 j}(K)(\approx \mathbf{Z}) \rightarrow \pi_{8 j}(K O)(\approx \mathbf{Z})$ is a multiplication by 2 , so is the morphism $E_{2}^{8 b,-8 b}(1)\left(\approx \mathbf{Z}_{4}\right) \longrightarrow E_{2}^{8 b,-8 b}(2)\left(\approx \mathbf{Z}_{4}\right)$. Therefore $2^{4 b} \bar{\lambda}_{k}$ is trivial in $F^{8 b} / F^{8 b+1}$.

Consider (ii). As in (i), we have an element $\omega_{3} \in \widetilde{K O}\left(L_{8 b}^{k}=S^{8 b} \vee L_{8 b+1}^{k}\right)$ such that $p^{*}\left(\omega_{3}\right)=2^{4 b-1} \bar{\lambda}_{k}$ and the restriction of $\omega_{3}$ on $S^{8 b}$ is $2 a_{2} g_{1} \in \widetilde{K O}\left(S^{8 b}\right)$ for some odd integer $a_{2}$. By Lemma 2.5, $2 \omega_{3}$ is trivial over $L_{8 b+1}^{k}$, so we can choose the desired $\eta_{2}$ and $a_{2}$.

For (iii), notice that by Lemma 2.4 , the bundle $2^{4 b+1} \lambda_{k}$ is stably trivial over $L^{8 b+3}$, and is of order 4 in $\widetilde{K O}\left(L^{8 b+4}\right)$. It follows by a similar argument and the fact that $L_{8 b+4}^{k}=S^{8 b+4} \vee L_{8 b+5}^{k}$.

Theorem 2.7. Suppose $n \not \equiv 0(\bmod 4)$, and $m \geq n+4$.

(i) The Adams operation $\Psi^{3}$ satisfies $\Psi^{3}(x)=3^{2 j} x$ for $x \in K O^{-4 j}\left(L_{n}^{m}\right)$.

(ii) $K O^{-4 j}\left(L_{n}^{m}\right) \approx \mathbf{Z} / 2^{a(m+4 j, n+4 j-1)} \oplus \mathbf{Z} / 2^{b(m+4 j, n+4 j-1)}$

where $h(u)=[u / 4]+[(u+4) / 8]+[(u+7) / 8]$, while $a(u, v)$ and $b(u, v)$ are defined by

$$
\begin{aligned}
& a(u, v)=h(u)-[(v+1) / 4]-[(v+6) / 8]-[(v+1) / 8], \\
& b(u, v)=[u / 8]+[(u+6) / 8]-[(v+7) / 8]-[(v+5) / 8],
\end{aligned}
$$

satisfying $a(u, v) \geq b(u, v)$.

(iii) There is an element in $K O^{4 k}\left(L_{4 k-1}^{4 k+t}\right)$ of order $2^{c(t)}$ (the maximum), where

$$
c(t)= \begin{cases}4 l+2 & \text { if } t=8 l \\ 4 l+3 & \text { if } t=8 l+1,8 l+2,8 l+3 \\ 4 l+5 & \text { if } t=8 l+4,8 l+5,8 l+6,8 l+7 .\end{cases}
$$

Proof. Consider (i). Let $\left\{E_{r}^{p, q}, d_{r}\right\}$ be the AHSS for $K O^{*}\left(L_{m}^{m+k}\right)$. Since each element of $E_{2}^{p, q}$ survives to $E_{\infty}$ when $p+q \equiv 0(\bmod 4)$, the morphism

$$
K O^{-4 j}\left(L_{n}^{m}\right) \rightarrow K O^{-4 j}\left(L^{m}\right)
$$

is injective when $n \not \equiv 0(\bmod 4)$ and $m>n$. Thus (i) follows from [14, Lemma 4.2].

Part (ii) is from [14, Theorem $2(1)]$. Here note that $a(m+8, n+8)=a(m, n)$, $b(m+8, n+8)=b(m, n)$, and $b(m+4 j, n+4 j-1) \geq 0$ when $m \geq n+4$. The formula is even true for integer $j<0$.

Consider (iii). First we have

$$
\begin{aligned}
h(u) & = \begin{cases}u / 2 & \text { if } u \equiv 0,2,6(\bmod 8), \\
(u+1) / 2 & \text { if } u \equiv 1,5(\bmod 8), \\
(u-1) / 2 & \text { if } u \equiv 3,7(\bmod 8), \\
u / 2+1 & \text { if } u \equiv 4(\bmod 8)\end{cases} \\
& = \begin{cases}\phi(u) & \text { if } u \neq \equiv 2,3(\bmod 8), \\
\phi(u)-1 & \text { otherwise }\end{cases}
\end{aligned}
$$

By (ii), there is an element of order $2^{a(t,-2)}$ in $K O^{4 k}\left(L_{4 k-1}^{4 k+t}\right)$. Let $c(t)=a(t,-2)$. Then

$$
c(t)=h(t)+2= \begin{cases}\phi(t)+2 & \text { if } t \not \equiv 2,3(\bmod 8), \\ \phi(t)+1 & \text { otherwise }\end{cases}
$$


Lemma 2.8. Let $4 j+1 \geq 4 n+2 k$.

(i) The Adams operation on $K O_{4 j+1}\left(L_{4 n+1}^{4 n+2 k}\right)$ satisfies $\left(\Psi^{3}-1\right)(g)=4 g$. If $2 k-1<8$, then $4 g=0$ for $g \in K O_{4 j+1}\left(L_{4 n+1}^{4 n+2 k}\right)$. If $2 k-1 \geq 8$, then

$$
K O_{4 j+1}\left(L_{4 n+1}^{4 n+2 k}\right) \approx \mathbf{Z} / 2^{[(2 k+3) / 4]} \oplus A \oplus B,
$$

where $A \approx \mathbf{Z}_{2}$ if $j-n$ even, and 0 otherwise; and

$$
B= \begin{cases}\mathbf{Z}_{2} & \text { if } 4(j-n)-2 k \equiv 0,2(\bmod 8), \\ 0 & \text { otherwise }\end{cases}
$$

(ii) Suppose $k \geq 5$. Then each element $x \in K O_{4 j+1}\left(L_{4 n+1}^{4 n+2 k}\right)$ is divisible by 4 if $x$ is in $F^{4 n+1}$ and is in the image of the projection

$$
p_{*}: K O_{4 j+1}\left(L_{-\infty}^{4 n+2 k}\right) \rightarrow K O_{4 j+1}\left(L_{4 n+1}^{4 n+2 k}\right) .
$$

Proof. Part (i) is from [14, Theorem 2 (2) and (5.10) (1)], where the summand $A$ is from the bottom cell, and $B$ is from the cells near the top. For (ii), consider the composite $L_{-\infty}^{4 n+2 k} \longrightarrow L_{4 n^{\prime}+1}^{4 n+2 k} \longrightarrow L_{4 n+1}^{4 n+2 k}, n^{\prime}<n$. With an appropriate $n^{\prime}$ and using (i), we can assume that the image of

$$
K O_{4 j+1}\left(L_{-\infty}^{4 n+2 k}\right) \longrightarrow K O_{4 j+1}\left(L_{4 n+1}^{4 n+2 k}\right)
$$

is in $\mathbf{Z} / 2^{[(2 k+3) / 4]} \oplus B$. Since $k \geq 5,[(2 k+3) / 4] \geq 3$. Then (ii) follows from the fact that $x$ is of $C W$-filtration $4 n+1$.

\section{Proofs of theorems}

It is known that if $J\left((A-B) \bar{\lambda}_{k}\right)=0$ over $L^{k}$, then $L_{2 A}^{2 A+k}$ and $L_{2 B}^{2 B+k}$ are Thom spaces of $J$-equivalent vector bundles, therefore a stable equivalence exists. We call a stable equivalence obtained in this way an equivalence (5), to distinguish from those four equivalences in Theorem 3.2.

Proof of Theorem 1.1. When $k>0, L_{n}^{n+k}$ is not $S$-coreducible if $n$ is odd, and $L_{n}^{n+k}$ is not $S$-reducible if $n+k$ is even. So (i) is immediate when $k=1$. By Steenrod operations, and noting that by Lemma 2.5 the order of $\lambda_{k}$ is 2 when $k=2$, 3, we see that $L_{n}^{n+k} \sim L_{m}^{m+k}$ iff $\nu(n-m) \geq 2$ when $k=2,3$.

Consider (ii) and (iii). Assume that $L_{n}^{n+k}$ is $S$-coreducible (if $S$-reducible use $S$-duality). By [3, Proposition 2.8], $L_{n}^{n+k}$ is $S$-coreducible iff $J\left(\frac{n}{2} \bar{\lambda}_{k}\right)=0$, where $J$ is the standard homomorphism $\widehat{K O}(-) \rightarrow J(-)$. By [8, Theorem 2.1], the order of $J\left(\bar{\lambda}_{k}\right)$ is

$$
\begin{cases}4 l & \text { if } k=8 l \\ 4 l+1 & \text { if } k=8 l+1,8 l+2,8 l+3 \\ 4 l+3 & \text { if } k=8 l+4,8 l+5,8 l+6,8 l+7 .\end{cases}
$$

Hence we have (ii). For (iii), if $L_{n}^{n+k} \sim L_{m}^{m+k}$, then $L_{n}^{n+k}$ is $S$-coreducible iff $L_{m}^{m+k}$ is $S$-coreducible, so the inequalities follow from (ii). Conversely, if the inequalities in (iii) are satisfied, then an equivalence (5) exists.

By Adams operation $\Psi^{5}$ on $K^{*}(-)$, we have

Lemma 3.1 ([11, Theorem 1.1 and p. 292]). If $L_{n}^{n+k} \sim L_{m}^{m+k}$, then

$$
\nu(n-m) \geq[(n+k) / 2]-[n / 2] .
$$

Proof of the necessity in Theorems 1.2 and 1.3. The necessity in Theorem 1.3 (ii) follows from Lemma 5.11 (ii) (or [13, Theorem 2 (2), p. 699]). By Steenrod op- 
erations, we have $\nu(n-m) \geq 3$ when $4 \leq k \leq 7$. The proof of the necessity in Theorem 1.3 (i) follows from the following observations.

Suppose $n \equiv 0(\bmod 4)$. By Lemma $3.1, \nu(n-m) \geq 4 l+3$ when $k-8 l=7,6$; $\nu(n-m) \geq 4 l+2$ when $k-8 l=5,4 ; \nu(n-m) \geq 4 l+1$ when $k-8 l=3,2$; $\nu(n-m) \geq 4 l$ when $k-8 l=1,0$.

Suppose $n \equiv 1(\bmod 4)$. By Lemma 3.1, $\nu(n-m) \geq 4 l+4$ when $k-8 l=7$; $\nu(n-m) \geq 4 l+3$ when $k-8 l=6,5 ; \nu(n-m) \geq 4 l+2$ when $k-8 l=4,3$; $\nu(n-m) \geq 4 l+1$ when $k-8 l=2,1 ; \nu(n-m) \geq 4 l$ when $k=8 l$.

Suppose $n \equiv 2(\bmod 4)$. By Lemma 2.1, Theorem 2.7 (i), (iii), and using $S$ duality, we have $\nu(n-m) \geq 4 l+4$ when $k-8 l=6$, thus when $k-8 l=7$; $\nu(n-m) \geq 4 l+2$ when $k-8 l=2$, thus when $k-8 l=5,4,3$. By Lemma 3.1, $\nu(n-m) \geq 4 l$ when $k-8 l=1,0$.

Suppose $n \equiv 3(\bmod 4)$. By Lemma 2.1, Theorem 2.7 (i), (iii), we have $\nu(n-m) \geq$ $4 l+4$ when $k-8 l=5$, thus when $k-8 l=7,6 ; \nu(n-m) \geq 4 l+2$ when $k-8 l=2$, thus when $k-8 l=3,4$. By Lemma 3.1, $\nu(n-m) \geq 4 l+1,4 l$ when $k-8 l=1,0$.

Theorem 3.2. Suppose $b \geq 0$ in (1), and $b \geq 1$ in (2)-(4). Assume none of the stunted lens spaces in question is $S$-coreducible or $S$-reducible.

(1) $L_{4 n}^{4 n+8 b+7} \sim L_{4 m}^{4 m+8 b+7}$ if $\nu(4 n-4 m) \geq 4 b+3$

(2) $L_{4 n}^{4 n+8 b+5} \sim L_{4 m}^{4 m+8 b+5}$ if $\nu(4 n-4 m) \geq 4 b+2$ (or $4 b+3$ when Theorem 1.3 (ii) is satisfied);

(3) $L_{4 n}^{4 n+8 b+3} \sim L_{4 m}^{4 m+8 b+3}$ if $\nu(4 n-4 m) \geq 4 b+1$;

(4) $L_{4 n}^{4 n+8 b+1} \sim L_{4 m}^{4 m+8 b+1}$ if $\nu(4 n-4 m) \geq 4 b$ (or $4 b+1$ when Theorem 1.3 (ii) is satisfied).

We call a stable equivalence given by Theorem $3.2(\mathrm{j})$, an equivalence (j). Assuming the above theorem, we can complete the sufficiency in Theorems 1.2, 1.3.

Proof of the sufficiency in Theorem 1.2. (a) $n \equiv 0(\bmod 4)$. If $k=7$, use an equivalence (1). By removing from both sides of an equivalence $L_{4 A}^{4 A+7} \sim L_{4 B}^{4 B+7}$, the top cell, the top two cells, top three cells respectively, we have the desired equivalences for $k=6,5,4$.

(b) $n \equiv 1(\bmod 4)$. If $k=7$, use an equivalence (4) to get an equivalence $L_{4 A}^{4 A+9} \sim L_{4 B}^{4 B+9}$, then remove the top cell and the bottom cell from both sides of that equivalence. If $k=6$, remove the bottom cell from both sides of an equivalence $L_{4 A}^{4 A+7} \sim L_{4 B}^{4 B+7}$ given by an equivalence (1); if $k=5,4$, remove respectively the top cell, the top two cells from both sides of an equivalence $L_{4 A+1}^{4 A+7} \sim L_{4 B+1}^{4 B+7}$.

(c) $n \equiv 2(\bmod 4)$. If $k=7,6$, use an equivalence $(5)$; if $k=5$, remove the bottom two cells from both sides of an equivalence $L_{4 A}^{4 A+7} \sim L_{4 B}^{4 B+7}$; if $k=4$, remove the top cell from both sides of an equivalence $L_{4 A+2}^{4 A+7} \sim L_{4 B+2}^{4 B+7}$.

(d) $n \equiv 3(\bmod 4)$. If $k=7$, use an equivalence $(4)$ and $S$-duality to get an equivalence $L_{4 A+2}^{4 A+11} \sim L_{4 B+2}^{4 B+11}$, then remove the bottom cell and the top cell; if $k=6,5$, remove respectively the top cell, the top two cells from both sides of an equivalence $L_{4 A+3}^{4 A+10} \sim L_{4 B+3}^{4 B+10}$; if $k=4$, remove the bottom three cells from both sides of an equivalence $L_{4 A}^{4 A+7} \sim L_{4 B}^{4 B+7}$ given by an equivalence (1).

Proof of the sufficiency in Theorem 1.3. (a) $n \equiv 0(\bmod 4)$. If $k \equiv 7,6(\bmod 8)$, use equivalences $(1)$; if $k \equiv 5,4(\bmod 8)$, use equivalences $(2)$; if $k \equiv 3,2(\bmod 8)$, use equivalences $(3)$; if $k \equiv 1,0(\bmod 8)$, use equivalences $(4)$. 
(b) $n \equiv 1(\bmod 4)$. If $k \equiv 7(\bmod 8)$, use an equivalence $(4)$, then remove the bottom cell from both sides of that equivalence; if $k \equiv 6(\bmod 8)$, use an equivalence $(1)$ and $S$-duality; if $k \equiv 5(\bmod 8)$, remove the top cell from both sides of an equivalence given by the case $k \equiv 6(\bmod 8)$; if $k \equiv 4(\bmod 8)$, use an equivalence (2), then remove the bottom cell from both sides of that equivalence; if $k \equiv 3(\bmod 8)$, remove the top cell from both sides of an equivalence given by the case $k \equiv 4(\bmod 8)$; if $k \equiv 2(\bmod 8)$, use an equivalence $(3)$ and $S$-duality; if $k \equiv 1(\bmod 8)$, remove the top cell from both sides of an equivalence given by the case $k \equiv 2(\bmod 8)$; if $k \equiv 0(\bmod 8)$, use an equivalence $(4)$ and remove the bottom cell from both sides of that equivalence.

(c) $n \equiv 2(\bmod 4)$. If $k \equiv 7,6(\bmod 8)$, use equivalences $(5)$; if $k \equiv 5,4(\bmod 8)$, use equivalences $(2)$ and $S$-duality; if $k \equiv 3,2(\bmod 8)$, use equivalences $(5)$; if $k \equiv 1,0(\bmod 8)$, use equivalences $(4)$ and $S$-duality.

(d) $n \equiv 3(\bmod 4)$. If $k \equiv 7(\bmod 8)$, use an equivalence $(4)$ and $S$-duality, then remove the bottom cell from both sides of that equivalence; if $k \equiv 6,5(\bmod 8)$, remove respectively the top cell, the top two cells from both sides of an equivalence given by the case $k \equiv 7(\bmod 8)$; if $k \equiv 4(\bmod 8)$, use an equivalence $(2)$ and $S$-duality; if $k \equiv 3(\bmod 8)$, remove the top cell from both sides of an equivalence given by the case $k \equiv 4(\bmod 8)$; if $k \equiv 2(\bmod 8)$, use an equivalence $(5)$; if $k \equiv 1(\bmod 8)$, use an equivalence $(3)$ and $S$-duality, then remove the bottom cell from both sides of that equivalence; if $k \equiv 0(\bmod 8)$, use an equivalence $(4)$ and $S$-duality.

Proof of Theorem 3.2. Maps will be stable here.

(1) $L_{4 n}^{4 n+8 b+7} \sim L_{4 m}^{4 m+8 b+7}$ when $\nu(4 n-4 m) \geq 4 b+3$.

Let $\lambda=\lambda_{8 b+7}$. Note that $L_{4 n}^{4 n+8 b+7}=T(2 n \lambda), L_{4 n-4 m}^{4 n-4 m+8 b+7}=T(2(n-m) \lambda)$ and $L_{4 m}^{4 m+8 b+7}=T(2 m \lambda)$. Since $\Delta^{*}(2(n-m) \lambda \times 2 m \lambda)=2 n \lambda$, where $\Delta: L^{8 b+7} \rightarrow L^{8 b+7} \times$ $L^{8 b+7}$ is the diagonal map, we have a map

$$
T(2 n \lambda) \rightarrow T(2(n-m) \lambda \times 2 m \lambda)=T(2(n-m) \lambda) \wedge T(2 m \lambda),
$$

namely a map $f_{1}: L_{4 n}^{4 n+8 b+7} \rightarrow L_{4 n-4 m}^{4 n-4 m+8 b+7} \wedge L_{4 m}^{4 m+8 b+7}$. We may assume $4(n-m) \geq$ 0 . Since $\nu(4 n-4 m) \geq 4 b+3$, by Theorem 2.6 (iii), there is a stable vector bundle $\eta$ over $S^{8 b+4}$ such that $p^{*}(2 \eta)=2(n-m) \lambda$, where $p$ is the projection $L^{8 b+7} \rightarrow L_{8 b+4}^{8 b+7}=S^{8 b+4} \vee L_{8 b+5}^{8 b+7} \rightarrow S^{8 b+4}$. Thus we have a map

$$
f_{2}: L_{4 n-4 m}^{4 n-4 m+8 b+7}=T(2(n-m) \lambda) \rightarrow T(2 \eta)=S^{4 n-4 m} \cup_{2 \beta} e^{4 n-4 m+8 b+4},
$$

where $\beta$ is the image of $\bar{\eta}$ under the $J$-homomorphism $\pi_{8 b+4}(B O) \rightarrow \pi_{8 b+3}^{s}$, thus $\beta=a \beta_{8 b+3}$ for some integer $a$. Let $f_{3}$ be the composite

$L_{4 n}^{4 n+8 b+7} \stackrel{f_{1}}{\longrightarrow} L_{4 n-4 m}^{4 n-4 m+8 b+7} \wedge L_{4 m}^{4 m+8 b+7} \stackrel{f_{2} \wedge 1}{\longrightarrow}\left(S^{4 n-4 m} \cup_{2 \beta} e^{4 n-4 m+8 b+4}\right) \wedge L_{4 m}^{4 m+8 b+7}$.

Taking a $C W$-approximation of $f_{3}$, we get a map

$$
f_{4}: L_{4 n}^{4 n+8 b+7} \rightarrow \Sigma^{4(n-m)}\left(L_{4 m}^{4 m+8 b+7} \cup_{2 \beta \vee 2 \beta \vee 2 \beta}\left(e^{4 m+8 b+4} \vee C M \vee e^{4 m+8 b+7}\right)\right)
$$

where $C M$ is the cone on the $\bmod 4$ Moore space $M=S^{4 m+8 b+4} \cup_{4} e^{4 m+8 b+5}$. Since $L_{4 m}^{4 m+8 b+7}$ is not $S$-reducible, by Theorem 1.1, we have $\nu(4 m+8 b+8) \leq 4 b+3$. Thus by Lemma 6.2, the top part of the 3-part wedge splits off. By Lemma 5.7, the next to top part $C M$ also splits off. Consequently we have a projection

$$
\begin{gathered}
r: \Sigma^{4(n-m)}\left(L_{4 m}^{4 m+8 b+7} \cup_{2 \beta \vee 2 \beta \vee 2 \beta}\left(e^{4 m+8 b+4} \vee C M \vee e^{4 m+8 b+7}\right)\right) \\
\rightarrow \Sigma^{4(n-m)}\left(L_{4 m}^{4 m+8 b+7} \cup_{2 \beta} e^{4 m+8 b+4}\right),
\end{gathered}
$$


which is the identity on the part $L_{4 m}^{4 m+8 b+7}$. Let

$$
f_{5}=r f_{4}: L_{4 n}^{4 n+8 b+7} \longrightarrow \Sigma^{4(n-m)}\left(L_{4 m}^{4 m+8 b+7} \cup_{2 \beta} e^{4 m+8 b+4}\right) .
$$

Taking the $S$-dual of $f_{5}$, we have a map

$$
g: X=\left(S^{-4 m-8 b-5} \vee L_{-4 m-8 b-8}^{-4 m-2}\right) \cup_{(2 \beta \vee \alpha)} e^{-4 m-1} \rightarrow \Sigma^{4(n-m)} L_{-4 n-8 b-8}^{-4 n-1}
$$

Since $L_{4 n}^{4 n+8 b+7}$ is not $S$-coreducible, $\nu(4 n) \leq 4 b+3$. By Lemma 6.2, the composite

$$
S^{-4 m-2} \stackrel{2 \beta}{\longrightarrow} S^{-4 m-8 b-5} \stackrel{g}{\longrightarrow} \Sigma^{4(n-m)} L_{-4 n-8 b-8}^{-4 n-1}
$$

is null, so $g: L_{-4 m-8 b-8}^{-4 m-2} \rightarrow \Sigma^{4(n-m)} L_{-4 n-8 b-8}^{-4 n-1}$ extends to

$$
L_{-4 m-8 b-8}^{-4 m-1} \rightarrow \Sigma^{4(n-m)} L_{-4 n-8 b-8}^{-4 n-1}
$$

which is an equivalence below the top cell. We claim it is an equivalence.

If $\nu(4 n)=\nu(4 m)$, then it follows from Lemma 5.11 (ii). Since $\nu(4 n-4 m) \geq 4 b+3$, the only possible case that $\nu(4 n) \neq \nu(4 m)$ was when

$$
\nu(4 n)>\nu(4 m) \geq 4 b+3 \text { or } \nu(4 m)>\nu(4 n) \geq 4 b+3,
$$

which would imply one of the original stunted lens spaces is $S$-coreducible.

(2) $L_{4 n}^{4 n+8 b+5} \sim L_{4 m}^{4 m+8 b+5}$ when $\nu(4 n-4 m) \geq 4 b+2$.

If both $\nu(4 n)$ and $\nu(4 m) \geq 4 b+3$, then an equivalence follows from case $(1)$. Suppose $\nu(4 n) \leq 4 b+2$. By the diagonal map and then a $C W$-approximation, we have a map

$$
f_{1}: L_{4 n}^{4 n+8 b+5} \rightarrow \Sigma^{4 n-4 m}\left(L_{4 m}^{4 m+8 b+5} \cup_{\beta \vee \beta} e^{4 m+8 b+4} \vee e^{4 m+8 b+5}\right)
$$

where $\beta=a \beta_{8 b+3}$ for some integer $a$. By Lemma 6.1 , the top part of the 2-part wedge splits off, and we have a map $f_{2}: L_{4 n}^{4 n+8 b+5} \rightarrow \Sigma^{4 n-4 m}\left(L_{4 m}^{4 m+8 b+5} \cup_{\beta} e^{4 m+8 b+4}\right)$. Let

$$
g:\left(S^{-4 m-8 b-5} \vee L_{-4 m-8 b-6}^{-4 m-2}\right) \cup_{\beta \vee \alpha} e^{-4 m-1} \rightarrow \Sigma^{4(n-m)} L_{-4 n-8 b-6}^{-4 n-1}
$$

be the dual of $f_{2}$. By Lemmas 6.4 (iii), 6.5 (ii), and with an argument as in the previous case, we see that $g: L_{-4 m-8 b-6}^{-4 m-2} \rightarrow \Sigma^{4(n-m)} L_{-4 n-8 b-6}^{-4 n-1}$ extends to a map $g_{5}: L_{-4 m-8 b-6}^{-4 m-1} \rightarrow \Sigma^{4(n-m)} L_{-4 n-8 b-6}^{-4 n-1}$ which is an equivalence below the top. If $\nu(4 n)=\nu(4 m)$, then an equivalence follows from Lemma 5.11 (ii). Since $\nu(4 n-4 m) \geq 4 b+2$, the only possible case that $\nu(4 n) \neq \nu(4 m)$ is when

$$
\nu(4 n)>\nu(4 m) \geq 4 b+2 \quad \text { or } \quad \nu(4 m)>\nu(4 n) \geq 4 b+2 .
$$

If $\nu(4 n)$ and $\nu(4 m)$ both $>4 b+2$, then the one of the original stunted lens spaces was $S$-coreducible.

If $\nu(4 n)$ (or $\nu(4 m))$ is $4 b+2$, then the condition of (2) requires $\nu(4 n-4 m) \geq 4 b+3$. So (3.3) is ruled out.

(4) $L_{4 n}^{4 n+8 b+1} \sim L_{4 m}^{4 m+8 b+1}$ when $\nu(4 n-4 m) \geq 4 b$.

First assume $b>1$. By taking the diagonal map and then a $C W$-approximation, we have a map

$$
f_{1}: L_{4 n}^{4 n+8 b+1} \rightarrow \Sigma^{4 n-4 m}\left(L_{4 m}^{4 m+8 b+1} \cup_{2 \beta \vee 2 \beta \vee \beta^{\prime}}\left(e^{4 m+8 b} \vee e_{1}^{4 m+8 b+1} \vee e_{2}^{4 m+8 b+1}\right)\right)
$$

where $\beta, \beta^{\prime}$ are respectively multiples of $\beta_{8 b-1}, \beta_{8 b}$. By Lemma 6.1 , the top part $e_{1}^{4 n+8 b+1}$ splits off, thus we have a map

$$
f_{2}: L_{4 n}^{4 n+8 b+1} \rightarrow \Sigma^{4 n-4 m}\left(L_{4 m}^{4 m+8 b+1} \cup_{2 \beta \vee \beta^{\prime}}\left(e^{4 m+8 b} \vee e^{4 m+8 b+1}\right)\right)
$$


Let $f_{3}: L_{4 n}^{4 n+8 b-1} \rightarrow \Sigma^{4 n-4 m} L_{4 m}^{4 m+8 b+1}$ and $f^{\prime \prime \prime}: L_{4 n}^{4 n+8 b-1} \rightarrow \Sigma^{4 n-4 m} L_{4 m}^{4 m+8 b}$ be the appropriate restrictions of $f_{2}$.

We can view $L_{4 n}^{4 n+8 b+1}$ as the complex

$$
L_{4 n}^{4 n+8 b-1} \cup_{\alpha_{1} \vee \alpha_{2}}\left(e^{4 n+8 b} \vee e^{4 n+8 b+1}\right) .
$$

Let $\xi$ be the real Hopf bundle over $P^{8 b}$. Since $\nu(4 n-4 m) \geq 4 b$, both $4(n-m) \xi$ and $2(n-m) \lambda_{8 b-1}$ are trivial. Thus we have an equivalence $f: P_{4 n}^{4 n+8 b} \rightarrow \Sigma^{4(n-m)} P_{4 m}^{4 m+8 b}$ by the $S$-coreducibility of $P_{4(n-m)}^{4(n-m)+8 b}$ and the map

$$
P_{4 n}^{4 n+8 b} \rightarrow P_{4(n-m)}^{4(n-m)+8 b} \wedge P_{4 m}^{4 m+8 b} \rightarrow \Sigma^{4 n-4 m} P_{4 m}^{4 m+8 b}
$$

Consider the diagram

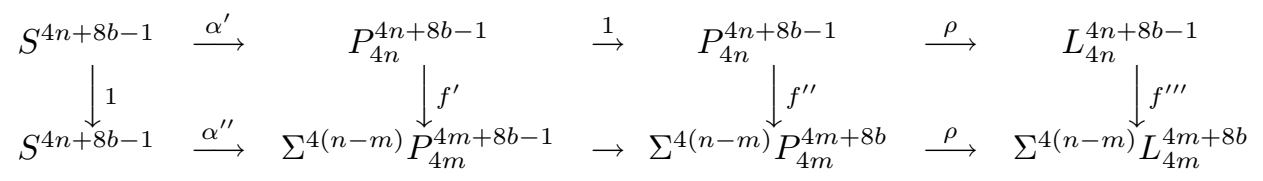

where $\alpha^{\prime}, \alpha^{\prime \prime}$ are the attaching maps for the top cells of $P_{4 n}^{4 n+8 b}$ and $\Sigma^{4(n-m)} P_{4 m}^{4 m+8 b}$, and $f^{\prime}, f^{\prime \prime}$ are restrictions of $f$. Let $\alpha_{1}^{\prime}$ be the attaching map for the top cell of $\Sigma^{4(n-m)} L_{4 m}^{4 m+8 b}$. Then both $\alpha_{1}$ and $\alpha_{1}^{\prime}$ are the attaching maps for the even dimensional cells, by Lemma 2.2 (i) or the $C W$-structure given by [21, p. 91], we have $\alpha_{1}=\rho \alpha^{\prime}$ and $\alpha_{1}^{\prime}=\rho \alpha^{\prime \prime}$. Thus the composite of the bottom row is homotopic to

$$
S^{4 n+8 b-1} \stackrel{\alpha_{1}}{\longrightarrow} L_{4 n}^{4 n+8 b-1} \stackrel{f^{\prime \prime \prime}}{\longrightarrow} \Sigma^{4(n-m)} L_{4 m}^{4 m+8 b}
$$

which is null-homotopic because $f^{\prime \prime}$ extends to $f$. Here the commutativity of the middle and the right squares is immediate, while the left follows from the existence of $f$. Hence $f_{3}$ extends to a map $f_{4}: L_{4 n}^{4 n+8 b} \rightarrow \Sigma^{4 n-4 m} L_{4 m}^{4 m+8 b+1}$, which is an equivalence on $(4 n+8 b)$-skeletons, by $\mathrm{Sq}^{2}$ and Lemma 5.11 (i).

On the other hand, it is well known that the first morphism in the exact sequence

$$
\begin{aligned}
& \pi_{4 n+8 b}\left(S^{4 n+8 b-1}\right) \stackrel{2 \beta}{\longrightarrow} \pi_{4 n+8 b}\left(\Sigma^{4(n-m)}\left(L_{4 m}^{4 m+8 b+1} \cup_{\beta^{\prime}} e^{4 m+8 b+1}\right)\right) \\
& \stackrel{i_{*}}{\longrightarrow} \pi_{4 n+8 b}\left(\Sigma^{4(n-m)}\left(L_{4 m}^{4 m+8 b+1} \cup_{2 \beta \vee \beta^{\prime}}\left(e^{4 m+8 b}\right) \vee e^{4 m+8 b+1}\right)\right)
\end{aligned}
$$

is 0 since $\beta$ is a multiple of $\beta_{8 b-1}$.

Now that $i_{*}\left(f_{4} \alpha_{2}\right)=f_{2} \alpha_{2}=0$, we conclude that $f_{4} \alpha_{2}$ is null-homotopic. Thus $f_{4}$ extends to a map

$$
g: L_{4 n}^{4 n+8 b+1} \rightarrow \Sigma^{4(n-m)}\left(L_{4 m}^{4 m+8 b+1} \cup_{\beta^{\prime}} e^{4 m+8 b+1}\right)
$$

such that $g^{*}$ on $H^{*}\left(-; \mathbf{Z}_{2}\right)$ is an isomorphism on the part $\Sigma^{4(n-m)} L_{4 m}^{4 m+8 b+1}$.

Taking the $S$-dual of $g$ as in the final stage of (1) or (2), using Lemmas 5.10,6.3 5.11 (ii), we have a desired equivalence.

Assume $b=1$. By $S$-duality, it suffices to show $L_{4 n+2}^{4 n+11} \sim L_{4 m+2}^{4 m+11}$. If both $\nu(4 m+12)$ and $\nu(4 n+12) \geq 5$, then an equivalence (5) follows because neither of the spaces is $S$-reducible implies $\nu(4 m+12)=\nu(4 n+12)=5$. So we may assume $2 \leq \nu(4 m+12) \leq 4$

Case (a). Suppose both $n$ and $m$ are odd. Taking the diagonal and then a $C W$-approximation, we have a map

$$
f_{1}: L_{4 n+2}^{4 n+11} \rightarrow \Sigma^{4(n-m)}\left(L_{4 m+2}^{4 m+11} \cup_{2 \beta_{7} \vee 2 \beta_{7} \vee \beta^{\prime}}\left(e^{4 m+10} \vee e_{1}^{4 m+11} \vee e_{2}^{4 m+11}\right)\right)
$$


Since $3 \leq \nu(4 m+12) \leq 4$, by Lemmas 6.3 (ii), 5.10 the top parts $e_{1}^{4 n+11} \vee e_{2}^{4 n+11}$ split off, and we have a map

$$
f_{2}: L_{4 n+2}^{4 n+11} \rightarrow \Sigma^{4(n-m)}\left(L_{4 m+2}^{4 m+11} \cup_{2 \beta_{7}} e^{4 m+10}\right) .
$$

Just as in (1), taking the $S$-dual and using Lemma 6.1 , we have a desired equivalence.

Case (b). Suppose both $n$ and $m$ are even. By $S$-duality, the statement that $L_{4 n+2}^{4 n+11} \sim L_{4 m+2}^{4 m+11}$ when $\nu(4(n-m)) \geq 4$ is equivalent to that $L_{8 A+4}^{8 A+13} \sim L_{8 B+4}^{8 B+13}$ when $\nu(8(A-B)) \geq 4$. By Case (a) and $S$-duality, we have an equivalence $g_{0}$ : $L_{8(A-B)}^{8(A-B)+9} \rightarrow \Sigma^{8(A-B)-2^{5}} L_{2^{5}}^{2^{5}+9}$. Since $2^{3} \lambda_{9}$ is trivial over $L^{7}$ and $L_{2^{5}}^{2^{5}+9}=T\left(2^{4} \lambda_{9}\right)$, we have a map

$$
g_{1}: L_{2^{5}}^{2^{5}+9} \rightarrow S^{2^{5}} \cup_{4 \beta_{7}} e^{2^{5}+8}
$$

of Thom spaces. Let $g_{2}$ be the composite

$$
\begin{aligned}
L_{8 A+4}^{8 A+13} \rightarrow & L_{8(A-B)}^{8(A-B)+9} \wedge L_{8 B+4}^{8 B+13} \stackrel{g_{0} \wedge 1}{\longrightarrow} \Sigma^{8(A-B)-2^{5}} L_{2^{5}}^{2^{5}+9} \wedge L_{8 B+4}^{8 B+13} \\
& \stackrel{g_{1} \wedge 1}{\longrightarrow}\left(S^{8(A-B)} \cup_{4 \beta_{7}} e^{8(A-B)+8}\right) \wedge L_{8 B+4}^{8 B+13} .
\end{aligned}
$$

Taking a $C W$-approximation, we have a map

$$
g_{3}: L_{8 A+4}^{8 A+13} \rightarrow \Sigma^{8(A-B)}\left(L_{8 B+4}^{8 B+13} \cup_{4 \beta_{7} \vee 4 \beta_{7}}\left(e^{8 B+12} \vee e^{8 B+13}\right)\right) .
$$

By Lemma 5.2, the top part of the 2-part wedge splits off, so we have map

$$
g_{4}: L_{8 A+4}^{8 A+13} \rightarrow \Sigma^{8(A-B)}\left(L_{8 B+4}^{8 B+13} \cup_{4 \beta_{7}} e^{8 B+12}\right)
$$

inducing an isomorphism on $H^{*}\left(-; \mathbf{Z}_{2}\right)$ when restricted on the part $\Sigma^{8(A-B)} L_{8 B+4}^{8 B+13}$. Let

$$
g_{5}:\left(S^{-8 B-13} \vee L_{-8 B-14}^{-8 B-6}\right) \cup_{4 \beta_{7} \vee \alpha} e^{-8 B-5} \rightarrow \Sigma^{8(A-B)} L_{-8 A-14}^{-8 A-5}
$$

be the $S$-dual of $g_{4}$. Since $g_{5} \mid S^{-8 B-13}$ is the degree one map into the $(-8 B-13)$ cell of $\Sigma^{8(A-B)} L_{-8 A-14}^{-8 A-5}$, we have $\left(g_{5} \mid S^{-8 B-13}\right)_{*}\left(4 \beta_{7}\right)=0$ by Lemma 5.10 . Thus $g_{5} \mid L_{-8 B-14}^{-8 B-6}$ extends to a map $L_{-8 B-14}^{-8 B-5} \rightarrow \Sigma^{8(A-B)} L_{-8 A-14}^{-8 A-5}$, which is an equivalence by $\mathrm{Sq}^{4}$ and Lemma 5.11 (ii).

(3) $L_{4 n}^{4 n+8 b+3} \sim L_{4 m}^{4 m+8 b+3}$ when $\nu(4 n-4 m) \geq 4 b+1$.

Suppose $b \geq 2$. By $S$-duality, we can assume that both $n$ and $m$ are even. As in (1), the diagonal map gives a map

$$
f_{1}: L_{4 n}^{4 n+8 b+3} \rightarrow L_{4(n-m)}^{4(n-m)+8 b+3} \wedge L_{4 m}^{4 m+8 b+3} .
$$

Since $\nu(4 n-4 m) \geq 4 b+1$, by Theorem 2.6 (ii), there is a stable vector bundle $\eta$ over $S^{8 b}$ such that $p^{*}(4 \eta)=2(n-m) \lambda_{8 b+3}$, where $p$ is the projection $L^{8 b+3} \rightarrow L_{8 b}^{8 b+3} \rightarrow S^{8 b}$. Thus we have a map

$$
f_{2}: L_{4(n-m)}^{4(n-m)+8 b+3} \rightarrow T(4 \eta)=S^{4(n-m)} \cup_{4 \beta} e^{4(n-m)+8 b},
$$

where $\beta=a \beta_{8 b-1}$ for some integer $a$. Let

$$
f_{3}=\left(f_{2} \wedge 1\right) f_{1}: L_{4 n}^{4 n+8 b+3} \rightarrow\left(S^{4(n-m)} \cup_{4 \beta} e^{4(n-m)+8 b}\right) \wedge L_{4 m}^{4 m+8 b+3} .
$$

Taking a $C W$-approximation of $f_{3}$, we have a map

$$
f_{4}: L_{4 n}^{4 n+8 b+3} \rightarrow \Sigma^{4 n-4 m}\left(L_{4 m}^{4 m+8 b+3} \cup_{4 \beta \vee 4 \beta \vee 4 \beta}\left(e^{4 m+8 b} \vee C M \vee e^{4 m+8 b+3}\right)\right)
$$


where $C M$ is the cone on the $\bmod 4$ Moore space $M=S^{4 m+8 b} \cup_{4} e^{4 m+8 b+1}$. By Lemma 6.3 (i), the top part of the 3-part wedge splits off. By Lemma 5.2, the next to top part also splits off. Thus we have a map

$$
f_{5}: L_{4 n}^{4 n+8 b+3} \rightarrow \Sigma^{4(n-m)}\left(L_{4 m}^{4 m+8 b+3} \cup_{4 \beta} e^{4 m+8 b}\right) .
$$

View $L_{4 n}^{4 n+8 b+3}=L_{4 n}^{4 n+8 b-1} \cup_{\alpha_{1} \vee \alpha_{2} \vee \alpha_{3}}\left(e^{4 n+8 b} \vee C N \vee e^{4 n+8 b+3}\right)$, where $C N$ is the cone on $N=S^{4 n+8 n} \cup_{4} e^{4 n+8 b+1}$. Let $f_{6}: L_{4 n}^{4 n+8 b-1} \rightarrow \Sigma^{4(n-m)} L_{4 m}^{4 m+8 b+3}$ be the map obtained by restricting $f_{5}$ on the $(4 n+8 b-1)$-skeleton.

As in (4), $f_{6} \alpha_{1}=0$ in homotopy. Since $\eta \beta_{8 b-1}=\beta_{8 b}$ by [16], and $4 \beta_{3}=\eta^{3}$, we have $4 \beta_{3} \beta_{8 b-1}=\eta^{3} \beta_{8 b-1}=0$. So the first morphism in the exact sequence

$$
\left[X, S^{4 n+8 b-1}\right] \stackrel{4 \beta_{8 b-1}}{\longrightarrow}\left[X, \Sigma^{4(n-m)} L_{4 m}^{4 m+8 b+3}\right] \stackrel{i_{*}}{\longrightarrow}\left[X, \Sigma^{4(n-m)}\left(L_{4 m}^{4 m+8 b+3} \cup_{4 \beta} e^{4 m+8 b}\right)\right]
$$

is null for $X=S^{4 n+8 b+2}$. It is also null for $X=N$ by Lemma 5.2. Thus both $f_{6} \alpha_{2}$ and $f_{6} \alpha_{3}$ are null-homotopic, and $f_{6}$ extends to a map over $L_{4 n}^{4 n+8 b+3}$ which can be required to be an equivalence by Lemma 5.11 .

Suppose $b=1$. By $S$-duality, we can assume that both $n$ and $m$ are odd. Thus both $\nu(4 n+12)$ and $\nu(4 m+12) \geq 3$. Using Lemma 6.3 (ii), and as for the case $b \geq 2$, we can find an equivalence $f_{6}: L_{4 n}^{4 n+11} \rightarrow \Sigma^{4(n-m)} L_{4 m}^{4 m+11}$.

\section{J-HOMOLOGY AND COEXTENSION}

According to [5], the stable Adams operation $\psi^{3}: b o \rightarrow b o$ is defined and $\psi^{3}-1$ : $b o \rightarrow b o$ lifts to $\Sigma^{4} b s p$. Let $\theta: b o \rightarrow \Sigma^{4} b s p$ be the lift and $J$ its fibre. There is a long exact sequence

$$
\rightarrow b s p_{*+1}\left(\Sigma^{4} X\right) \stackrel{\omega}{\rightarrow} J_{*}(X) \rightarrow b o_{*}(X) \stackrel{\theta_{*}}{\rightarrow} b s p_{*}\left(\Sigma^{4} X\right) \rightarrow .
$$

Use the fact that $\pi_{t}(b o)=\pi_{t}(K O) \otimes \mathbf{Z}_{(2)}$ if $t \geq 0$, and is 0 otherwise; while $\pi_{t}\left(\Sigma^{4} b s p\right)=\pi_{t}(K O) \otimes \mathbf{Z}_{(2)}$ if $t \geq 4$, and is 0 otherwise, we can compute $b o_{*}(X)$ and $b s p_{*}\left(\Sigma^{4} X\right)$ and $\theta_{*}: b o_{*}(X) \rightarrow b s p_{*}\left(\Sigma^{4} X\right)$ in some cases. For example, if $X=P^{2 n}$, then both $b o_{4 j-1}(X)$ and $b s p_{4 j-1}\left(\Sigma^{4} X\right)$ are cyclic and $\theta_{*}$ sends a generator to $2^{\nu(j)} g$, where $g \in b s p_{4 j-1}\left(\Sigma^{4} X\right)$ is a generator, this is because $\left(\psi^{3}-1\right)(x)=$ $2^{\nu(j)+2} x\left(\bmod 2^{\nu(j)+3}\right)$ in $b o_{4 j-1}(X)$ and the projection $b_{s p} p_{4 j-1}\left(\Sigma^{4} X\right) \rightarrow b o_{4 j-1}(X)$ is injective with image divisible by 4 .

Let $J: \pi_{k}(S O) \rightarrow \pi_{k}^{s}$ be the standard $J$-homomorphism. By [18, Theorem 1.1.13], ImJ is cyclic with 2 -component $\mathbf{Z}_{(2)} /(2(k+1))$ when $k \equiv 3(\bmod 8)$, and $\mathbf{Z}_{2}$ when $k \equiv 0,1(\bmod 8)$, and the Hurewicz map $\pi_{k}^{s} \rightarrow \pi_{k}(J)$ is injective on ImJ. Putting $\beta_{8 b-1}, \beta_{8 b+3}, \beta_{8 b+1}$, generators of $\operatorname{ImJ}$, into the bottom cell, we get classes in $\pi_{*}\left(L_{n}^{n+k}\right)$.

Lemma 4.2. Let $b \geq 2$ (i), and $b \geq 1$ in (ii)-(iv).

(i) $2 \beta_{8 b-1}$ is null in $J_{4 A+8 b}\left(L_{4 A+1}^{4 A+10}\right)$ but not null in $J_{4 A+8 b}\left(L_{4 A+1}^{4 A+9}\right)$.

(ii) For odd $A, \beta_{8 b-1}$ is null in $J_{4 A+8 b-2}\left(P_{4 A-1}^{4 A+6}\right)$ but not null in $J_{4 A+8 b-2}\left(P_{4 A-1}^{4 A+5}\right)$.

(iii) $\beta_{8 b+3}$ is null in $J_{4 A+8 b+4}\left(L_{4 A+1}^{4 A+10}\right)$ but not null in $J_{4 A+8 b+4}\left(L_{4 A+1}^{4 A+9}\right)$.

(iv) If $A$ is even, then $\beta_{8 b+1}$ is null in $J_{4 A+8 b+2}\left(P_{4 A+1}^{4 A+8}\right)$ but not null in $J_{4 A+8 b+2}\left(P_{4 A+1}^{4 A+7}\right)$.

Proof. We just show (i) and (ii). The proofs for (iii) and (iv) are similar.

Consider (i). Since $b \geq 2$, there is a natural isomorphism $f_{*}: b o_{4 A+8 b+1}\left(L_{4 A+1}^{4 A+10}\right)$ $\approx b s p_{4 A+8 b+1}\left(\Sigma^{4} L_{4 A+1}^{4 A+10}\right)$. By Lemma 2.8 (i), the morphism

$$
\theta_{*}: b o_{4 A+8 b+1}\left(L_{4 A+1}^{4 A+10}\right) \rightarrow b s p_{4 A+8 b+1}\left(\Sigma^{4} L_{4 A+1}^{4 A+10}\right)
$$


in (4.1) satisfies $\theta_{*}(x)=4 f_{*}(x)(\bmod 8)$. Note that $2 \beta \in J_{4 A+8 b}\left(L_{4 A+1}^{4 A+10}\right)$ is from an element $z \in b s p_{4 A+8 b+1}\left(\Sigma^{4} L_{4 A+1}^{4 A+10}\right)$, that is from the bottom cell corresponding to the order 2 element $z^{\prime} \in E_{4 A+5,8 b-4}^{2}$ in the AHSS for $b s p_{*}\left(\Sigma^{4} L_{4 A+1}^{4 A+10}\right)$. Let $p$ be the projection as in Lemma 2.8 (ii). Since $z^{\prime}$ survives in the AHSS for $b s p_{*}\left(\Sigma^{4} L_{-\infty}^{4 A+10}\right)$, $z$ is in the image of $p_{*}$. By Lemma 2.8 (ii), $z$ is divisible by 4 and hence is hit by $\theta_{*}$. So $2 \beta$ is null in $J_{4 A+8 b}\left(L_{4 A+1}^{4 A+10}\right)$. However $2 \beta$ is not null in $J_{4 A+8 b}\left(L_{4 A+1}^{4 A+8}\right)$ because all elements of $b s p_{4 A+8 b+1}\left(\Sigma^{4} L_{4 A+1}^{4 A+8}\right)$ are of order $\leq 4$ by Lemma 2.8 (i), thus are not hit by $\theta_{*}$. Finally the $d_{2}$-differential on the unique nontrivial element $y$ of $E_{4 A+9,8(b-1)}^{2}=H_{4 A+9}\left(L_{4 A+3}^{4 A+9}, J_{8(b-1)}\right) \approx \mathbf{Z}_{2}$ in the AHSS for $J_{*}\left(L_{4 A+3}^{4 A+9}\right)$ is not zero. Thus $J_{4 A+8 b+1}\left(L_{4 A+3}^{4 A+8}\right) \rightarrow J_{4 A+8 b+1}\left(L_{4 A+3}^{4 A+9}\right)$ is surjective by exactness. This together with the fact that $2 \beta$ is nontrivial in $J_{4 A+8 b}\left(L_{4 A+1}^{4 A+8}\right)$ imply that the boundary $J_{4 A+8 b+1}\left(L_{4 A+3}^{4 A+9}\right) \rightarrow J_{4 A+8 b}\left(L_{4 A+1}^{4 A+2}\right)$ does not hit $2 \beta \in J_{4 A+8 b}\left(L_{4 A+1}^{4 A+2}\right)$, and (i) follows.

Consider (ii). Note that the composite

$$
b o_{4 A+8 b-1}\left(P_{4 A-2}^{4 A+5}\right) \stackrel{\theta_{*}}{\longrightarrow} \operatorname{bsp}_{4 A+8 b-1}\left(\Sigma^{4} P_{4 A-2}^{4 A+5}\right) \rightarrow b o_{4 A+8 b-1}\left(P_{4 A-2}^{4 A+5}\right)
$$

sends $x$ to $8 x$. Thus $\beta_{8 b-1}$ is not null in $J_{4 A+8 b-2}\left(P_{4 A-2}^{4 A+5}\right)$ because

$$
b o_{4 A+8 b-1}\left(P_{4 A-2}^{4 A+5}\right) \approx K O_{4 A+8 b-1}\left(P_{4 A-2}^{4 A+5}\right) \approx \mathbf{Z}_{8},
$$

which implies that $\beta_{8 b-1}$ is not hit by $\theta_{*}$. However, $b o_{4 A+8 b-1}\left(P_{4 A-2}^{4 A+6}\right) \approx \mathbf{Z}_{16}$, hence $\beta_{8 b-1}$ is hit by $\theta_{*}$.

Let $E_{r}(X)$ be the $E_{r}$ term in ASS for $\pi_{*}(X)$. Given $x \in \pi_{*}(X)$, let $A(x)$ be the Adams filtration of $x$. Let $A_{k}\left(\pi_{*}(X)\right)$ be the subgroup of $\pi_{*}(X)$ of classes of Adams filtrations $\geq k$.

Lemma 4.3. Let $n \geq a>b$. Let $I(n, a, b)$ be the image of the projection $J_{*}\left(L_{b}^{n}\right) \rightarrow$ $J_{*}\left(L_{a}^{n}\right)$.

(i) Suppose $x: S^{m} \rightarrow L_{a}^{n}$ satisfies $A(x) \geq N$ and $x \in I(n, a, b)$ when put into $J_{*}(-)$. If the projection $A_{N+1}\left(\pi_{m-1}\left(S^{a-1}\right)\right) \rightarrow J_{m-1}\left(S^{a-1}\right)$ is injective, and each nontrivial element of $E_{2}^{s, s+m-1}\left(S^{a-1}\right)$ survives to a homotopy class when $s \geq N+1$, then $x$ coextends to a map $z: S^{m} \rightarrow L_{a-1}^{n}$ with $A(z) \geq N$.

(ii) If moreover the projection $A_{N}\left(\pi_{m}\left(S^{a-1}\right)\right) \rightarrow J_{m}\left(S^{a-1}\right)$ is surjective, then the $z$ in (i) can be chosen to be in $I(n, a-1, b)$ when put into $J_{*}(-)$.

Proof. Consider (i). Here the idea is similar to [4, Lemma 6]. First the condition implies that the boundary $\partial: \pi_{m}\left(L_{a}^{n}\right) \rightarrow \pi_{m-1}\left(S^{a-1}\right)$ is null on $x$. By $S$-duality, it suffices to show $x_{0}: L_{-n-1}^{-a-1} \rightarrow S^{-m-1}$, the dual of $x$, extends to a map $x^{\prime}$ : $L_{-n-1}^{-a} \rightarrow S^{-m-1}$ with $A\left(x^{\prime}\right) \geq N$. Let $\alpha$ be the attaching map for the top cell of $L_{-n-1}^{-a}$. Since $x_{0}$ lifts to $E^{N}$, the $N$-stage of the stable Adams resolution of $S^{-m-1}$, and $A\left(x_{0} \alpha\right) \geq N+1$, the only way that the composite $x_{0} \alpha: S^{-a-1} \rightarrow E^{N}$ fails to be null-homotopic is that in the ASS for $\pi_{*}\left(S^{0}\right)$ there was a nontrivial differential $E_{r}^{s, s+m-a+1} \rightarrow E_{r}^{s+r, s+r+m-a}$ with $s<N<s+r$. But this is ruled out by the condition.

Consider (ii). There exists $y \in J_{m}\left(L_{b}^{n}\right)$ which is a coextension of the composite $S^{m} \stackrel{x}{\rightarrow} L_{a}^{n} \rightarrow J \wedge L_{a}^{n}$. So $p(y)-z$ pulls back to $J_{m}\left(S^{a-1}\right)$, where $p$ is the projection $L_{b}^{n} \rightarrow L_{a-1}^{n}$. Pick up an element $y^{\prime} \in \pi_{m}\left(S^{a-1}\right)$ with $A\left(y^{\prime}\right) \geq N$, and $p(y)-z=y^{\prime}$ in $J_{m}\left(S^{a-1}\right)$. Then $z+y^{\prime}$ is the desired coextension. 


\section{AdAMS Filtration}

By [17, Theorem 8.2], if $t \geq 11$, then $\beta_{t}$ has both the same Adams filtration in $\pi_{t}\left(S^{0}\right)$ and $J_{t}\left(S^{0}\right)$ except when $t=8 c-1$. As indicated in [4, p. 344], the composite $S^{2 N+8 c-2} \stackrel{\beta_{8 c-1}}{\longrightarrow} S^{2 N-1} \rightarrow P_{2 N-1}^{2 N}$ has Adams filtration $4 c-2$ for $c \geq 2$.

Lemma 5.1. Suppose $\nu(n+1)=i, 4 c-1 \leq i \leq 4 c+2$ and $c \geq 1$. Let $a=i-$ $4 c+1$. Suppose $e_{0}: S^{n} \rightarrow P_{n-8 c+2}^{n}$ is the degree one map given by the $S$-reducibility. Let $\iota$ generate $\pi_{n}\left(S^{n}\right)$, and $\partial: \pi_{n}\left(P_{n-8 c+2}^{n}\right) \rightarrow \pi_{n-1}\left(P_{n-8 c-7}^{n-8 c+1}\right)$ the boundary. Then $\partial\left(e_{0}\right)=x$, where

$$
x: S^{n-1} \stackrel{2^{a}}{\stackrel{\beta_{8 c-1}}{\longrightarrow}} S^{n-8 c} \rightarrow P_{n-8 c-7}^{n-8 c} \rightarrow P_{n-8 c-7}^{n-8 c+1} .
$$

Here the third map is the inclusion, the second is given by the $S$-reducibility.

Proof. Choose $m$ such that $\nu(m)=i$. Then $P_{m}^{m+8 c+7}=T(m \xi)$, where $\xi$ is the real Hopf bundle over $P^{8 c+7}$. Then $m \xi$ is stably trivial over $P^{8 c-1}$. As in Theorem 2.6 (i), there is a stable vector bundle $\eta^{\prime}$ over $S^{8 c}$ corresponding to a generator of $\widetilde{K O}\left(S^{8 c}\right) \otimes \mathbf{Z}_{(2)}$, such that $p^{*}\left(2^{a} \eta^{\prime}\right)=m \bar{\xi}$, where $p$ is the composite $P^{8 c+7} \rightarrow P_{8 c}^{8 c+7} \rightarrow S^{8 c}$ in which the last map is given by the $S$-coreducibility. So we have a map $g: P_{m}^{m+8 c+7} \rightarrow S^{m} \cup_{2^{a} \beta_{8 c-1}} e^{m+8 c}$ of Thom spaces such that $g^{*}$ is injective on $H^{*}\left(-; \mathbf{Z}_{2}\right)$. By $S$-duality we have a map $g: S^{n-8 c} \cup_{2^{a}} \beta_{8 c-1} e^{n} \rightarrow P_{n-8 c-7}^{n}$ such that $g_{*}$ is injective on $H_{*}\left(-; \mathbf{Z}_{2}\right)$. By the diagram,

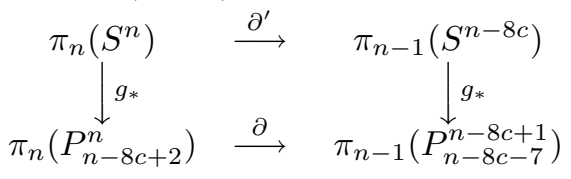

we have $\partial\left(e_{0}\right)=x$ with $e_{0}=g_{*}(\iota)$. This completes the proof.

Lemma 5.2. Let $M$ be the mod 4 Moore space with the bottom cell of dimension 0 . Then $A_{2}([M, M])=0$, and the identity map $1: M \rightarrow M$ is of order 4 .

Proof. Consider the exact sequence

$$
\left[S^{1}, M\right] \stackrel{4}{\longrightarrow}\left[S^{1}, M\right] \rightarrow[M, M] \rightarrow\left[S^{0}, M\right] \stackrel{4}{\longrightarrow}\left[S^{0}, M\right]
$$

derived from the cofibre sequence $S^{0} \stackrel{4}{\longrightarrow} S^{0} \rightarrow M \rightarrow S^{1} \stackrel{4}{\longrightarrow} S^{1}$.

Then in the ASS for $\pi_{*}(M)$, we have $E_{3}^{s, s}(M)=0$ by [18, Theorem 2.3.4, p. $63]$ when $s \geq 2$. So the only possible nontrivial elements of $A_{2}([M, M])$ are from $\left[S^{1}, M\right]$. By the exact sequence

$$
\left[S^{1}, S^{0}\right] \stackrel{4}{\longrightarrow}\left[S^{1}, S^{0}\right] \rightarrow\left[S^{1}, M\right] \rightarrow\left[S^{1}, S^{1}\right] \stackrel{4}{\longrightarrow}\left[S^{1}, S^{1}\right],
$$

we see that each nontrivial element of $E_{2}^{s, s+1}(M)$ supports a nontrivial $d_{2}$-differential when $s \geq 2$. This implies $A_{2}([M, M])=0$, and the order of $1: M \rightarrow M$ is of order $\leq 4$. Therefore $1: M \rightarrow M$ must be of order 4 because $H_{1}(M ; \mathbf{Z}) \approx \mathbf{Z}_{4}$.

The filtration 4 maps between real stunted projective spaces are studied in detail in [6], [15] and [17]. In the next lemma, $c, i$ and $\iota$ are the collapse map, the inclusion and the identity.

Lemma 5.3. (i) ([6, Prop. 2.1], [15, Theorem 3.3]) Let $m$ be odd, $n$ even, $m<n$. There is a stable map $g: P_{m+8}^{n+8} \rightarrow P_{m}^{n}$ such that $A(g)=4$, the composite $P_{m}^{n+8} \stackrel{c}{\longrightarrow}$ $P_{m+8}^{n+8} \stackrel{g}{\longrightarrow} P_{m}^{n} \stackrel{i}{\longrightarrow} P_{m}^{n+8}$ is $16 \iota$, and $g_{*}: K O_{8 k-1}\left(P_{m+8}^{n+8}\right) \rightarrow K O_{8 k-1}\left(P_{m}^{n}\right)$ is an isomorphism for all $k$. 
(ii) [17, Lemma 7.15] There is a map $g: P_{4 k+7} \rightarrow P_{4 k-1}$ such that $A(g)=4$, and $g_{*}: E_{2}^{s, t}\left(P_{4 k+7}\right) \rightarrow E_{2}^{s+4, t+4}\left(P_{4 k-1}\right)$ is an isomorphism in the ASS if $6 s>t+14-4 k$.

Lemma 5.4. There exists an unstable map $f: P_{8 n}^{8(n+1)+7} \rightarrow S^{8 n} \cup_{n \beta_{7}} e^{8(n+1)}$ whose restriction $S^{8 n} \rightarrow S^{8 n} \cup_{n \beta_{7}} e^{8(n+1)}$ induces a surjection on $\widetilde{K O}(-)$.

Proof. Since $P_{8}^{15}=T\left(8 \xi_{7}\right)$ and $8 \xi_{7}$ is trivial over $P^{7}$, we see $P_{8}^{15}=S^{8} \vee P_{9}^{15}$ in the unstable case. The bundle $8 \xi_{15}$ over $P^{15}$ is trivial on $P^{7}$, so we have an 8 dimensional bundle $\eta$ over $P_{8}^{15}$. Let $\eta^{\prime}$ be the restriction of $\eta$ on $S^{8}$. The composite

$$
P^{15} \longrightarrow P_{8}^{15}=S^{8} \vee P_{9}^{15} \longrightarrow S^{8}
$$

in the unstable case, induces a map

$$
f: T\left(8 n \xi_{15}\right)=P_{8 n}^{8 n+15} \longrightarrow T\left(n \eta^{\prime}\right)=e^{8 n} \cup_{n \beta_{7}} e^{8(n+1)}
$$

of Thom spaces, which is of degree one on the bottom cell.

Then the surjection follows from the fact that $K O^{1}\left(S^{8(n+1)}\right)=0$ in the exact sequence below

$$
K O^{0}\left(S^{8 n} \cup_{a \beta_{7}} e^{8(n+1)}\right) \rightarrow K O^{0}\left(S^{8 n}\right) \rightarrow K O^{1}\left(S^{8(n+1)}\right)
$$

Let $X$ be a $C W$-complex, $X^{n}$ its $n$-skeleton. Let $X_{n+1}^{n+k}=X^{n+k} / X^{n}$.

Lemma 5.5. Let $b$ be an integer. Suppose both $f$ and $g$ make the diagram

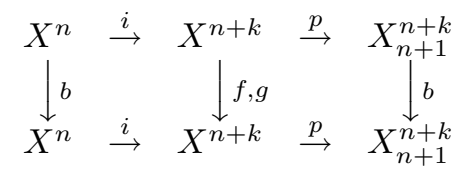

commute, where $i$ and $p$ are respectively the inclusion and the projection. Then $h=$ $f-g: X^{n+k} \rightarrow X^{n+k}$ factors as $X^{n+k} \stackrel{p}{\longrightarrow} X_{n+1}^{n+k} \rightarrow X^{n+k}$ and $X^{n+k} \rightarrow X^{n} \stackrel{i}{\longrightarrow} X^{n+k}$.

Proof. The first factoring follows from the fact that the restriction of $f-g$ on $X^{n}$ is null, while the second factoring is implied by $p(f-g)=0$.

Lemma 5.6. There exists a map $h: L_{2 k+1}^{2 k+2} \rightarrow L_{2 k+1}^{2 k+2}$ factoring as $L_{2 k+1}^{2 k+2} \rightarrow S^{2 k+1} \rightarrow$ $L_{2 k+1}^{2 k+2}$ such that $2-h: L_{2 k+1}^{2 k+2} \rightarrow L_{2 k+1}^{2 k+2}$ factors as $L_{2 k+1}^{2 k+2} \rightarrow P_{2 k+1}^{2 k+2} \stackrel{\rho}{\longrightarrow} L_{2 k+1}^{2 k+2}$.

Proof. There is a map $\epsilon$ such that the diagram

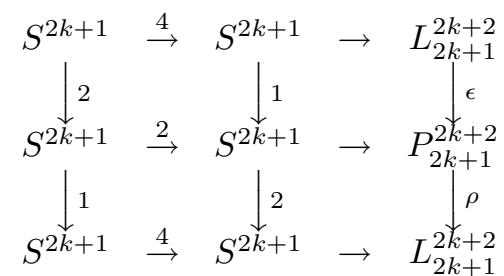

commutes. By Lemma 5.5, the map $h=2-\epsilon \rho$ is the desired.

Lemma 5.7. Let $\beta$ generate ImJ on the $(8 b+3)$-stem. Let $N=e^{4 m+1} \cup_{4} e^{4 m+2}$. If $\nu(4 m+8 b+8) \leq 4 b+2$, then the composite

$$
x: \Sigma^{8 b+3} N \stackrel{\beta \wedge 2}{\longrightarrow} N \rightarrow L_{4 m}^{4 m+8 b+7}
$$

is null-homotopic, where the second map is the inclusion to $L_{4 m}^{4 m+2}=S^{4 m} \vee N$. 
Proof. By Lemma 5.6, the map $N \stackrel{2}{\rightarrow} N \rightarrow L_{4 m}^{4 m+8 b+7}$ factors as $N \rightarrow P_{4 m}^{4 m+8 b+7} \stackrel{\rho}{\longrightarrow}$ $L_{4 m}^{4 m+8 b+7}$ up to a map $N \rightarrow S^{4 m+1} \rightarrow L_{4 m}^{4 m+8 b+7}$, where the second map is the degree one map to the $(4 m+1)$-cell. Thus by [4, Prop. 4] and Lemma 6.1 , it is null.

Lemma 5.8. Let $i=\nu(n+1)$.

(i) If $3 \leq i \leq 4 b+1$, then in $A S S$ for $\pi_{*}\left(P_{n-8 b-6}^{n}\right)$ elements in $E_{2}^{s, s+n-1}\left(P_{n-8 b-6}^{n}\right)$ of Adams filtrations $\geq 4 b$ are hit by Adams differentials.

(ii) If $3 \leq i \leq 4 b$, then in ASS for $\pi_{*}\left(P_{n-8 b-6}^{n}\right)$ elements in $E_{2}^{s, s+n-1}\left(P_{n-8 b-6}^{n}\right)$ of Adams filtrations $\geq 4 b-1$ are hit by Adams differentials.

Proof. We just show (i). The proof for (ii) is similar. In ASS for $\pi_{*}\left(P_{n-8 b-6}^{n-8 b+1}\right)$ the chart for $E_{2}^{s, s+n-1}\left(P_{n-8 b-6}^{n-8 b+1}\right)$ is as follows.

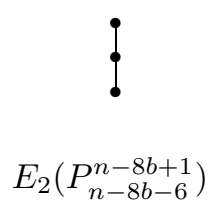

where the bottom class corresponds to $s=4 b-1$. The chart for $E_{2}\left(P_{n-8 b-6}^{n-8 b+1}\right)$ can be obtained by using the pre-spectral sequence (PSS, [16, p. 26]) converging to $E_{2}\left(P_{n-8 b-6}^{n-8 b+1}\right)$ with

$$
E_{1}^{s, t}=\Sigma_{k=-1}^{6} \operatorname{Ext}_{A}^{s, t}\left(H^{*}\left(S^{n-8 b-k}\right), \mathbf{Z}_{2}\right),
$$

and repeatedly using [16, table 8.1], the Adams periodicity, [2, Lemma 2.6.1], and [18, Theorem 2.3.4, p. 63].

Case $b=1$. Let $\partial$ be the boundary $\pi_{n}\left(S^{n}\right) \rightarrow \pi_{n-1}\left(P_{n-14}^{n-1}\right)$. Then by Lemma $5.1, \partial(\iota)$ is the class

$$
x: S^{n-1} \stackrel{2^{a} \beta_{7}}{\longrightarrow} S^{n-8} \rightarrow P_{n-14}^{n-8} \rightarrow P_{n-14}^{n-7}
$$

when put into $P_{n-14}^{n-1}$, where $a=i-3$. Let $y=2^{2-a} x$. Then $y$ is of Adams filtration $\geq 3$. Since $y$ is of Adams filtration 4 when put into $J_{n-1}\left(P_{n-14}^{n-7}\right)$, by the chart for $E_{2}^{s, s+n-1}\left(P_{n-14}^{n-7}\right), y$ is of Adams filtration 4 . This shows the case $b=1$.

Case $b \geq 2$. Suppose (i) holds for $b$. We wish to show (i) holds for $b+1$.

If $3 \leq \nu(n+1) \leq 4 b+1$, then it follows from the filtration 4 map $g: P_{n-8 b-6}^{n+1} \rightarrow$ $P_{n-8(b+1)-6}^{n-7}$ and the fact that the filtration $4 b$ element of $E_{2}^{4 b, 4 b+n-1}\left(P_{n-8 b-6}^{n+1}\right)$ is hit by an Adams differential. So we may assume $4 b+2 \leq \nu(n+1) \leq 4(b+1)+1$.

Part A. Assume (i) for $b=2$. Suppose $i=4 b+2$. Let $\Sigma P_{m}^{m+8(b+1)+6}$ be the $S$-dual of $P_{n-8(b+1)-6}^{n}$. By Lemma 5.4, there is an unstable map $f$ : $P_{8 b}^{8(b+1)+6} \rightarrow S^{8 b} \cup_{a \beta} e^{8(b+1)}$ and a stable vector bundle $\eta$ over $S^{8 b} \cup_{a \beta} e^{8(b+1)}$ such that $f^{*}(\eta)$ is a 4-multiple of a generator in $\widetilde{K O}\left(S^{8 b}\right)$ when restricted on the bottom cell $S^{8 b}$. So $(f p)^{*}(\eta)=(m / 2) \xi$, where $p$ is the projection $P^{8(b+1)+6} \rightarrow P_{8 b}^{8(b+1)+6}$. Thus there is a map of Thom spaces $f_{0}: P_{m / 2}^{m / 2+8(b+1)+6} \rightarrow T(\eta)$. Taking the diagonal and then a $C W$-approximation, we have a composite

$$
\begin{aligned}
& P_{m}^{m+8(b+1)+6} \stackrel{f_{1}}{\longrightarrow}\left(P_{m / 2}^{m / 2+8(b+1)+6} \wedge P_{m / 2}^{m / 2+8(b+1)+6}\right)^{(m+8(b+1)+6)} \\
& \stackrel{f_{2}}{\longrightarrow}(T(\eta) \wedge T(\eta))^{(m+8(b+1)+6)}
\end{aligned}
$$


where the restriction of $f_{2}$ on $P_{m / 2}^{m / 2+8(b+1)+6} \wedge S^{m / 2}$ or $S^{m / 2} \wedge P_{m / 2}^{m / 2+8(b+1)+6}$ is exactly $f_{0}$ as given above, and

$$
(T(\eta) \wedge T(\eta))^{(m+8(b+1)+6)} / S^{m}=\Sigma^{m / 2}\left(T(\eta) / S^{m / 2} \vee T(\eta) / S^{m / 2}\right)
$$

since $b \geq 2$. This observation is important to $(5.9)$.

Let

$$
\partial_{0}: \pi_{n}\left(S^{n}\right) \rightarrow \pi_{n-1}\left(\Sigma^{(n+1) / 2} P_{(n+1) / 2-8(b+1)-7}^{(n+1) / 2-2}\right)
$$

and

be boundaries.

$$
\partial_{1}: \pi_{n}\left(S^{n}\right) \rightarrow \pi_{n-1}\left(P_{n-8(b+1)-6}^{n-1}\right)
$$

Let $g_{1}$ and $g_{0}$ be the $S$-duals of $f_{1}$ and $f_{0}, \alpha_{1}=\partial_{0}(\iota)$. By $S$-duality, and as in Lemma 5.1, we have

$$
\partial_{1}(\iota)=2 g_{1 *}\left(\alpha_{1}\right) .
$$

In general, let $a=i-4 b-1$, and consider the composite

$$
P_{m}^{m+8(b+1)+6} \rightarrow P_{m / 2^{a}}^{m / 2^{a}+8(b+1)+6} \wedge \ldots \wedge P_{m / 2^{a}}^{m / 2^{a}+8(b+1)+6} \rightarrow T(\eta) \wedge \ldots \wedge T(\eta),
$$

where $P_{m / 2^{a}}^{m / 2^{a}+8(b+1)+6} \wedge \ldots \wedge P_{m / 2^{a}}^{m / 2^{a}+8(b+1)+6}$ is the smash product of $2^{a}$ copies of $P_{m / 2^{a}}^{m / 2^{a}+8(b+1)+6}$. Taking a $C W$-approximation and repeating the preceding argument, we have

$$
\partial_{1}(\iota)=2^{a} g_{1 *}\left(\alpha_{1}\right)
$$

where $\alpha_{1}=\partial_{0}(\iota)$, and $\partial_{0}: \pi_{n}\left(S^{n}\right) \rightarrow \pi_{n-1}\left(\Sigma^{n+1-(n+1) / 2^{a}} P_{(n+1) / 2^{a}-8(b+1)-7}^{(n+1) / 2^{a}-2}\right)$.

Let $x=2^{a} g_{1 *}\left(\alpha_{1}\right)$. Then $2^{4-a} x=2^{4} g_{1 *}\left(\alpha_{1}\right)$. By induction, $\alpha_{1}$ is of Adams filtration $4 b$ when projected to $P_{(n+1) / 2^{a}-8 b-7}^{(n+1) / 2^{a}-2}$, so $g_{1_{*}}\left(\alpha_{1}\right)$ is of Adams filtration $4 b$ when projected to $P_{n-8 b-6}^{n-1}$. Thus by the filtration 4 map again, we see that filtration $\geq 4(b+1)$ elements of $E_{2}^{s, s+n-1}\left(P_{n-8(b+1)-6}^{n}\right)$ are hit by Adams differentials. Moreover $\partial_{1}(\iota)$ is of Adams filtration $4(b+1)$ when $i=4(b+1)$.

Part B. Consider (i) for $b=2$. First assume $\nu(m)=5$. As before we have an unstable map $f: P_{8}^{22} \rightarrow S^{8} \cup_{\beta_{7}} e^{16}$ and a stable vector bundle $\eta$ over $S^{8} \cup_{\beta_{7}} e^{16}$, such that $(f p)^{*}(\eta)=(m / 2) \xi$. Let $f_{0}: P_{m / 2}^{m / 2+22} \rightarrow T(\eta)$ be a map of the Thom spaces. Taking the diagonal and then a $C W$-approximation, we have

$$
P_{m}^{m+22} \stackrel{f_{1}}{\longrightarrow}\left(P_{m / 2}^{m / 2+22} \wedge P_{m / 2}^{m / 2+22}\right)^{(m+22)} \stackrel{f_{2}}{\longrightarrow}(T(\eta) \wedge T(\eta))^{(m+22)} .
$$

Since $\pi_{7}\left(S^{0}\right)$ is generated by $\beta_{7}, \nu(m / 2) \geq 4$ and $\left(f_{0}\right)^{*}$ is injective on $H^{*}\left(-; \mathbf{Z}_{2}\right)$, we see that $a^{\prime}$ is odd in the following

$$
T(\eta) / S^{m / 2}=S^{m / 2+8} \cup_{a^{\prime} \beta_{7}} e^{m / 2+16}
$$

by $\mathrm{Sq}^{8}$. This implies that the attaching map $\alpha^{\prime}$ indicated below

$$
(T(\eta) \wedge T(\eta))^{(m+22)} / S^{m}=\Sigma^{m / 2}\left(\left(T(\eta) / S^{m / 2} \vee T(\eta) / S^{m / 2}\right) \cup_{\alpha^{\prime}} e^{m / 2+16}\right),
$$

is null. Thus

$$
(T(\eta) \wedge T(\eta))^{(m+22)} / S^{m}=\Sigma^{m / 2}\left(T(\eta) / S^{m / 2} \vee T(\eta) / S^{m / 2} \vee e^{m / 2+16}\right)
$$

So in this case time (5.9) becomes

$$
\partial_{1}(\iota)=2 g_{1 *}\left(\alpha_{1}\right)+x_{0},
$$


where $x_{0}$ is in the image of $\pi_{n-1}\left(S^{n-16}\right) \rightarrow \pi_{n-1}\left(P_{n-22}^{n-1}\right)$ induced by the degree one map from $S^{n-16}$ to the $(n-16)$-cell of $P_{n-22}^{n-1}$ with $A\left(x_{0}\right) \geq 4$. Here $\alpha_{1}=$ $\partial_{0}(\iota), \partial_{0}: \pi_{n}\left(S^{n}\right) \rightarrow \pi_{n-1}\left(\Sigma^{(n+1) / 2} P_{(n+1) / 2-23}^{(n+1) / 2-2}\right)$ and $\partial_{1}: \pi_{n}\left(S^{n}\right) \rightarrow \pi_{n-1}\left(P_{n-22}^{n-1}\right)$ are boundaries.

For $6 \leq i \leq 9$, we have

$$
\partial_{1}(\iota)=2^{a}\left(2 g_{1 *}\left(\alpha_{1}\right)+x_{0}\right)
$$

where $a=i-5$ and $\alpha_{1}=\partial_{0}(\iota): \pi_{n}\left(S^{n}\right) \rightarrow \pi_{n-1}\left(\sum^{n+1-(n+1) / 2^{a+1}} P_{(n+1) / 2^{a+1}-23}^{(n+1) / 2^{a+1}-2}\right.$. Thus

$$
\partial_{1}\left(2^{4-a} \iota\right)=2^{5} g_{1 *}\left(\alpha_{1}\right)+2^{4} x_{0}
$$

Since each element in $\pi_{n-1}\left(P_{n-22}^{n-15}\right)$ is of order $\leq 2^{4}$, we see $2^{4} x_{0}=0$. So as before, the proof follows from the filtration 4 map. Moreover if $i=9$, then $\partial_{1}(\iota)$ is of Adams filtration 8 .

Lemma 5.10. If $3 \leq \nu(4 n) \leq 4 b$, then both composites

$$
\begin{gathered}
S^{4 n-2} \stackrel{4 \beta_{8 b-1}}{\longrightarrow} S^{4 n-8 b-1} \longrightarrow L_{4 n-8 b-2}^{4 n-1}, \\
S^{4 n-2} \stackrel{\beta_{8 b}}{\longrightarrow} S^{4 n-8 b-2} \longrightarrow L_{4 n-8 b-2}^{4 n-1}
\end{gathered}
$$

are null-homotopic.

Proof. Notice that the boundary $\pi_{4 n-1}\left(S^{4 n-8 b}\right) \longrightarrow \pi_{4 n-2}\left(L_{4 n-8 b-2}^{4 n-8 b-1}\right)$ maps $\beta_{8 b-1}$ to

$$
S^{4 n-2} \quad \stackrel{\beta_{8 b} \vee 4 \beta_{8 b-1}}{\longrightarrow} \quad S^{4 n-8 b-2} \vee S^{4 n-8 b-1}=L_{4 n-8 b-2}^{4 n-8 b-1} .
$$

So in $\pi_{4 n-2}\left(L_{4 n-8 b-2}^{4 n-1}\right)$ both the composites in question are equal. The lemma follows from Lemma 5.8 .

We have repeatedly used the next lemma in section 3 .

Lemma 5.11. Suppose neither the top cell of $L_{n}^{n+k}$ nor of $L_{m}^{m+k}$ splits off. Let $g: L_{n}^{n+k} \rightarrow \Sigma^{n-m} L_{m}^{m+k}$ be an equivalence below the top cell.

(i) If $g$ is of odd degree on the top cell, then $g$ can be adjusted to an equivalence.

(ii) Suppose $\nu(m+k+1) \equiv \nu(n+k+1) \equiv 0(\bmod 4)$. Then $g$ is of odd degree on the top cell (hence an equivalence) $\Longleftrightarrow \nu(m+k+1)=\nu(n+k+1)$.

Proof. Consider (i). The case when $n+k$ is even is immediate. Assume $n+k$ is odd. Let $\alpha_{1}$ and $\alpha_{2}$ be the attaching maps for the top cells of $L_{n}^{n+k}$ and $\Sigma^{n-m} L_{m}^{m+k}$. Then $t \alpha_{2}=g_{*}\left(\alpha_{1}\right)$ for an odd $t$. Note that $\alpha_{2}$ is the image of an element $\alpha_{2}^{\prime} \in$ $\pi_{n+k-1}\left(\Sigma^{n-m} L^{m+k-1}\right)$ under the projection $\pi_{*}\left(\Sigma^{n-m} L^{m+k-1}\right) \rightarrow \pi_{*}\left(\Sigma^{n-m} L_{m}^{m+k-1}\right)$. Consider the diagram

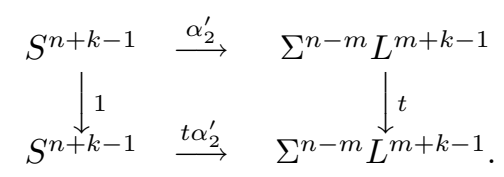

Since $t: \Sigma^{n-m} L^{m+k} \rightarrow \Sigma^{n-m} L^{m+k}$ is an equivalence, we can view $t \alpha_{2}^{\prime}$ (thus $t \alpha_{2}$ ) as the attaching map for the top cell of $\Sigma^{n-m} L^{m+k}$. Write $t \alpha_{2}$ as $\alpha_{2}$. Then $\alpha_{2}=g_{*}\left(\alpha_{1}\right)$, and (i) follows. 
Consider (ii). Let $0 \leq d<k$ be an integer satisfying

$$
k-d= \begin{cases}8 b & \text { if } \nu(n+k+1)=4 b, \\ 8 b-4 & \text { if } \nu(n+k+1)=4 b-1,4 b-2, \\ 8 b-7 & \text { if } \nu(n+k+1)=4 b-3 .\end{cases}
$$

Then by Theorem 1.1, $L_{n+d+1}^{n+k}$ is $S$-reducible but $L_{n+d}^{n+k}$ is not. Consider the map

$$
g^{\prime}: L_{n+d}^{n+k} \rightarrow \Sigma^{n-m} L_{m+d}^{m+k}
$$

induced by $g$, and the diagram

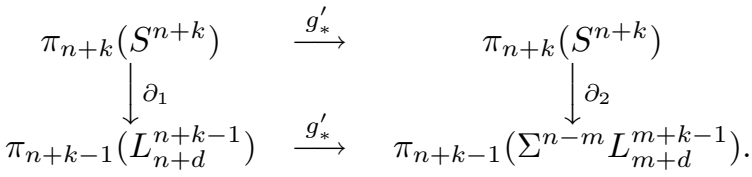

Suppose $\nu(m+k+1)=\nu(n+k+1)$. As in Lemma 5.1, then $\partial_{i}\left(e_{0}\right), i=1,2$, and $g_{*}^{\prime}\left(\partial_{1}\left(e_{0}\right)\right)$ are the classes corresponding to $\epsilon \beta_{k-1}$ for the same $\epsilon$. $L_{m}^{m+k}$ is not $S$-reducible implies that $\epsilon \beta_{k-1}$ is not null in $\pi_{n+k-1}\left(\Sigma^{n-m} L_{m}^{m+k-1}\right)$. Therefore $g^{\prime}$ (and hence $g$ ) must be of odd degree on the top cell.

If $g$ is an equivalence, then so is $g^{\prime}$. We have $g_{*}^{\prime}\left(\partial_{1}\left(e_{0}\right)\right)=\partial_{2}\left(e_{0}\right)$. This implies $\nu(m+k+1)=\nu(n+k+1)$.

\section{Triviality of $\tilde{\beta}_{t}$ IN $(1.4)$}

In this section, we study under some appropriate conditions the triviality of $\tilde{\beta}_{t}$ in (1.4) for $\beta_{t}=\beta_{8 b-1}$ or $\beta_{8 b+3}$.

Lemma 6.1. Let $b \geq 2$ in (i), and $b \geq 1$ in (ii). Let $\partial_{1}, \partial_{2}$ be respectively the boundaries $\pi_{*}\left(L_{4 A+3}^{4 A+10}\right) \rightarrow \pi_{*-1}\left(L_{4 A}^{4 A+2}\right), \pi_{*}\left(L_{4 A+2}^{4 A+10}\right) \rightarrow \pi_{*-1}\left(L_{4 A}^{4 A+1}\right)$.

(i) Let $2^{e+1}$ be the order of $\beta_{8(b-1)-1}$. There is an integer $i$ such that $e-1 \leq i \leq e$ and $2^{i} \beta_{8(b-1)-1}$ coextends to a map $x_{1}: S^{4 A+1+8 b} \rightarrow L_{4 A+3}^{4 A+10}$ satisfying $A\left(x_{1}\right) \geq$

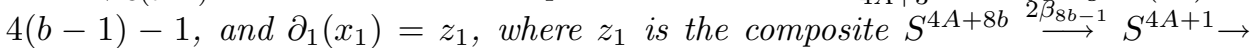
$L_{4 A}^{4 A+2}$.

(ii) There is an integer $i$ with $1 \leq i \leq 2$ such that $2^{i} \beta_{8 b-5}$ coextends to a map $x_{2}: S^{4 A+8 b+5} \rightarrow L_{4 A+2}^{4 A+10}$ satisfying $A\left(x_{2}\right) \geq 4 b-2$, and $\partial_{2}\left(x_{2}\right)=z_{2}$, where $z_{2}$ is the composite

$$
S^{4 A+8 b+4} \stackrel{\beta_{8 b+3}}{\longrightarrow} S^{4 A+1} \rightarrow L_{4 A}^{4 A+1} .
$$

(iii) Both composites $S^{4 A+8 b} \stackrel{z_{1}}{\longrightarrow} L_{4 A}^{4 A+2} \rightarrow L_{4 A}^{4 A+10}$ and $S^{4 A+8 b+4} \stackrel{z_{2}}{\longrightarrow} L_{4 A}^{4 A+1} \rightarrow$ $L_{4 A}^{4 A+10}$ are null-homotopic.

Proof. Part (iii) is just a corollary of (i) and (ii).

Consider (i). Let $\beta=\beta_{8 b-1}$. By Lemma 4.2 (i), $2 \beta$ is null in $J_{4 A+8 b}\left(L_{4 A+1}^{4 A+10}\right)$ but not null in $J_{4 A+8 b}\left(L_{4 A+1}^{4 A+9}\right)$. So the only possible element in $J_{4 A+8 b+1}\left(L_{4 A+3}^{4 A+10}\right)$ that might hit $2 \beta \in J_{*}\left(L_{4 A+1}^{4 A+2}\right)$ under the boundary

$$
\partial^{\prime}: J_{4 A+8 b+1}\left(L_{4 A+3}^{4 A+10}\right) \rightarrow J_{4 A+8 b}\left(L_{4 A+1}^{4 A+2}\right)
$$

is from the top cell of $L_{4 A+3}^{4 A+10}$, that is, there is an element $x \in J_{4 A+8 b+1}\left(L_{4 A+3}^{4 A+10}\right)$ that is from a nontrivial element

$$
u_{0} \in E_{4 A+10,8(b-1)-1}^{2}=H_{4 A+10}\left(L_{4 A+3}^{4 A+10} ; J_{8(b-1)-1}\right)
$$


in AHSS satisfying $\partial^{\prime}(x)=2 \beta$ in $J_{4 A+8 b}\left(L_{4 A+1}^{4 A+2}\right)$. Let $2^{i} \beta_{8(b-1)-1}: S^{4 A+8 b+1} \rightarrow$ $S^{4 A+10}$ correspond to $u_{0}$, then $e-1 \leq i \leq e$. Denote $2^{i} \beta_{8(b-1)-1}$ also by $u_{0}$.

We want to coextend $u_{0}$ to a map $x_{1}: S^{4 A+8 b+1} \rightarrow L_{4 A+3}^{4 A+10}$ with $A\left(x_{1}\right) \geq 4(b-$ 1) -1 . By [16, table 8.1], we see that when $-1 \leq t \leq 5$,

$$
A_{4(b-1)}\left(\pi_{8(b-1)+t}\left(S^{0}\right)\right) \rightarrow J_{8(b-1)+t}\left(S^{0}\right)
$$

is injective, and each nontrivial element of $E_{2}^{s, s+8(b-1)+t}\left(S^{0}\right)$ survives to a homotopy class in the image of $J$ if $s \geq 4(b-1)$; moreover the projection

$$
A_{4(b-1)-1}\left(\pi_{8(b-1)+t}\left(S^{0}\right)\right) \rightarrow J_{8(b-1)+t}\left(S^{0}\right)
$$

is surjective when $0 \leq t \leq 6$. By repeatedly applying Lemma 4.3 (i) and (ii), we have the desired coextension $x_{1}$.

Next we show $\partial_{1}\left(x_{1}\right)=z_{1}$ in $\pi_{4 A+8 b}\left(L_{4 A}^{4 A+2}\right)$. Consider the Adams spectral sequences for $\pi_{*}\left(L_{4 A+1}^{4 A+2}\right)$ and $\pi_{*}\left(L_{4 A}^{4 A+2}\right)$ and notice that

$$
E_{2}^{s, t}\left(L_{4 A+1}^{4 A+2}\right)=\sum_{k=0}^{1} \operatorname{Ext}^{s, t}\left(S^{4 A+1+k}\right)
$$

and

$$
E_{2}^{s, t}\left(L_{4 A}^{4 A+2}\right)=\sum_{0 \leq k \leq 2} \operatorname{Ext}^{s, t}\left(S^{4 A+1+k}\right) .
$$

By [16, table 8.1] and [18, Theorem 2.3.4, p.63], we get the charts for $E_{3}^{s, t}\left(L_{4 A+1}^{4 A+2}\right)$ and $E_{3}^{s, t}\left(L_{4 A}^{4 A+2}\right)$, where $s \geq 4(b-1)$ and $4 A+8 b \leq t-s \leq 4 A+8 b+1$, as follows.

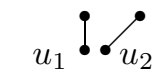

$E_{3}\left(L_{4 A+1}^{4 A+2}\right)$

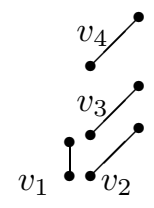

$E_{3}\left(L_{4 A}^{4 A+2}\right)$

where the bottom elements $u_{1}, u_{2}, v_{1}$ and $v_{2}$ are in the same position $(t-s, s)=$ $(4 A+8 b, 4(b-1)+1)$, and $u_{1}$ corresponds to $\beta_{8 b-1}$ into $\pi_{4 A+8 b}\left(L_{4 A+1}^{4 A+2}\right), u_{2}$ supports a nontrivial $d_{1}$-differential to $S^{4 A-1}$ in the ASS for $\pi_{*}\left(L_{4 A-2}^{4 A+2}\right)$. Also $v_{2}, v_{3}$ and $v_{4}$ support nontrivial $d_{1}$-differentials to $S^{4 A-2}$ in the ASS for $\pi_{*}\left(L_{4 A-2}^{4 A+2}\right)$, so those homotopy classes which $v_{2}, v_{3}$ and $v_{4}$ converge to, can not be in the image of $\partial_{1}$. Since $\partial^{\prime}\left(x_{1}\right)=2 \beta$ in $J_{4 A+8 b}\left(L_{4 A+1}^{4 A+2}\right)$ and $A\left(\partial_{1}\left(x_{1}\right)\right) \geq 4(b-1)$, we see that $\partial_{1}\left(x_{1}\right)$ corresponds to $2 v_{1}$, and hence $\partial_{1}\left(x_{1}\right)=z_{1}$ in $\pi_{4 A+8 b}\left(L_{4 A}^{4 A+2}\right)$ because $\eta\left(x_{1}\right)=0$, while $\eta$ is injective on the subgroup generated by classes corresponding to $v_{2}, v_{3}$ and $v_{4}$. Here $\eta=\beta_{1}$.

Consider (ii). Let $\beta=\beta_{8 b+3}$. By Lemma 4.2 (iii), $\beta$ is null in $J_{4 A+8 b+4}\left(L_{4 A+1}^{4 A+10}\right)$ but not null in $J_{4 A+8 b+4}\left(L_{4 A+1}^{4 A+9}\right)$. So the only possible element in $J_{4 A+8 b+4}\left(L_{4 A+3}^{4 A+10}\right)$ that might hit $\beta \in J_{*}\left(S^{4 A+1}\right)$ under the boundary

$$
\partial^{\prime}: J_{4 A+8 b+5}\left(L_{4 A+2}^{4 A+10}\right) \rightarrow J_{4 A+8 b+4}\left(S^{4 A+1}\right)
$$

is from the top cell of $L_{4 A+2}^{4 A+10}$, and there is an element $x \in J_{4 A+8 b+5}\left(L_{4 A+2}^{4 A+10}\right)$ that is from a nontrivial element $u_{0} \in E_{4 A+10,8(b-1)+3}^{2}=H_{4 A+10}\left(L_{4 A+2}^{4 A+10} ; J_{8(b-1)+3}\right)$ in 
AHSS satisfying $\partial^{\prime}(x)=\beta$ in $J_{4 A+8 b+4}\left(S^{4 A+1}\right)$. Let

$$
2^{i} \beta_{8(b-1)+3}: S^{4 A+8 b+5} \rightarrow S^{4 A+10} \rightarrow L_{4 A+10}^{4 A+12}
$$

correspond to $u_{0}, 1 \leq i \leq 2$. Consider the coextension of $2^{i} \beta_{8(b-1)+3}$ in a larger space $L_{4 A+6}^{4 A+12}$ since in ASS the $E_{3}$ chart for $L_{4 A+6}^{4 A+12}$ will be simple. Denote $2^{i} \beta_{8(b-1)+3}$ by $u_{0}$. By applying Lemma 4.3 (i) and (ii) repeatedly, we can coextend $u_{0}$ to a map $u_{1}: S^{4 A+8 b+5} \rightarrow L_{4 A+6}^{4 A+12}$ with $A\left(u_{1}\right) \geq 4 b-2$. In the ASS, the chart for $E_{3}^{s, t}\left(L_{4 A+6}^{4 A+12}\right)$ with $s \geq 4 b-2$ and $t-s=4 A+8 b+5$ is as follows.

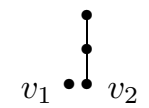

$$
E_{3}\left(L_{4 A+6}^{4 A+12}\right)
$$

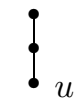

$$
E_{2}\left(L_{4 A}^{4 A+1}\right)
$$

where both $v_{1}$ and $v_{2}$ are in the position $(t-s, s)=(4 A+8 b+5,4 b-2), v_{1}$ corresponds to an order 2 class in homotopy that coextends to $J_{4 A+8 b+5}\left(L_{4 A+2}^{4 A+12}\right)$, and $v_{2}$ corresponds to the image of $2^{d-3} \beta_{8 b-1}$ under $\pi_{4 A+8 b+5}\left(S^{4 A+6}\right) \rightarrow \pi_{4 A+8 b+5}\left(L_{4 A+6}^{4 A+12}\right)$, where $2^{d}$ is the order of $\beta_{8 b-1}$. If $u_{1} \notin I(4 A+12,4 A+6,4 A+2)$ when put into $J_{*}(-)$, then $u_{1}=v_{1}+\epsilon v_{2}$ for some $\epsilon$. Here the homotopy class to which $v_{i}$ survives is also denoted by $v_{i}$. Choose a new $u_{1}$ to be $u_{1}-\epsilon v_{2}$. Then $u_{1}$ is a coextension of $u_{0}$ with $A\left(u_{1}\right)=4 b-2$ and $u_{1} \in I(4 A+12,4 A+6,4 A+2)$ when put into $J_{*}(-)$. Applying Lemma 4.3 (i) and (ii) repeatedly, we get the desired coextension $x_{2}$.

Finally in the ASS the charts for $E_{2}^{s, t}\left(L_{4 A}^{4 A+1}\right)$ and $E_{2}^{s, t}\left(S^{4 A+1}\right)$, where $s \geq 4 b-1$ and $t-s=4 A+8 b+4$, coincide as the right chart above, where $u$ is in the position $(t-s, s)=(4 A+8 b+4,4 b-1)$ surviving to $\beta_{8 b+3} \in \pi_{4 A+8 b+4}\left(S^{4 A+1}\right)$ or $z_{2} \in$ $\pi_{4 A+8 b+4}\left(L_{4 A}^{4 A+1}\right)$. Since $\partial^{\prime}\left(x_{2}\right)=\beta$ in $J_{4 A+8 b+4}\left(S^{4 A+1}\right)$ and $A\left(\partial_{2}\left(x_{2}\right)\right) \geq 4 b-1$, there must hold $\partial_{2}\left(x_{2}\right)=z_{2}$.

Lemma 6.2. (i) Let $x_{3}$ be the composite $S^{4 A+8 b+2} \stackrel{\beta_{8 b+3}}{\longrightarrow} S^{4 A-1} \rightarrow P_{4 A-4}^{4 A-1}$. If $\nu(4 A+8 b+4) \leq 4 b+2$, then $x_{3}$ is null-homotopic in $\pi_{4 A+8 b+2}\left(P_{4 A-4}^{4 A+8 b+3}\right)$.

(ii) Let $x_{4}$ be the composite $S^{4 A+8 b+2} \stackrel{2 \beta_{8 b+3}}{\longrightarrow} S^{4 A-1} \rightarrow L_{4 A-4}^{4 A-1}$. If $\nu_{2}(4 A+8 b+4) \leq$ $4 b+3$, then $x_{4}$ is null-homotopic in $\pi_{4 A+8 b+2}\left(L_{4 A-4}^{4 A+8 b+3}\right)$.

Proof. Let $\beta=\beta_{8 b+3}$. Consider (i). The case $\nu(4 A+8 b+4)<4 b+2$ is from [ 4 , Proposition 5]. Assume $\nu(4 A+8 b+4)=4 b+2$. Let $D P_{4 A-4}^{4 A+8 b+3}=\Sigma P_{n}^{n+8 b+7}$, where $\nu(n)=4 b+2$. Since $P_{n}^{n+8 b+7}=T(n \xi)$, where $\xi$ is the Hopf real line bundle over $P^{8 b+7}$, and $\nu(n)=4 b+2$, there is a stable vector bundle $\eta$ over $S^{8 b+4}$ corresponding to a generator of $\widetilde{K O}\left(S^{8 b+4}\right) \otimes \mathbf{Z}_{(2)}$ and $n \bar{\xi}=p^{*}(\eta)$, where $p$ is the projection $P^{8 b+7} \rightarrow P_{8 b+4}^{8 b+7} \rightarrow S^{8 b+4} \vee P_{8 b+5}^{8 b+7} \rightarrow S^{8 b+4}$. Note that $T(\eta)=S^{n} \cup_{\beta} e^{n+8 b+4}$. We have a map $f: P_{n}^{n+8 b+7} \rightarrow T(\eta)$ such that $f^{*}$ is injective on $H^{*}\left(-; \mathbf{Z}_{2}\right)$, and the induced map $P_{n+8 b+4}^{n+8 b+7} \rightarrow T(\eta) / S^{n}$ is precisely the projection $S^{n+8 b+4} \vee P_{n+8 b+5}^{n+8 b+7} \rightarrow S^{n+8 b+4}$. Thus by duality we have a map $g: S^{4 A-1} \cup_{\beta} e^{4 A+8 b+3} \rightarrow P_{4 A-4}^{4 A+8 b+3}$ such that $g_{*}$ is injective on $H_{*}\left(-; \mathbf{Z}_{2}\right)$ and the restriction of $g$ on $S^{4 A-1}$ is the map $S^{4 A-1} \rightarrow S^{4 A-1} \vee$ 
$P_{4 A-4}^{4 A-2}=P_{4 A-4}^{4 A-1}$. There is a diagram

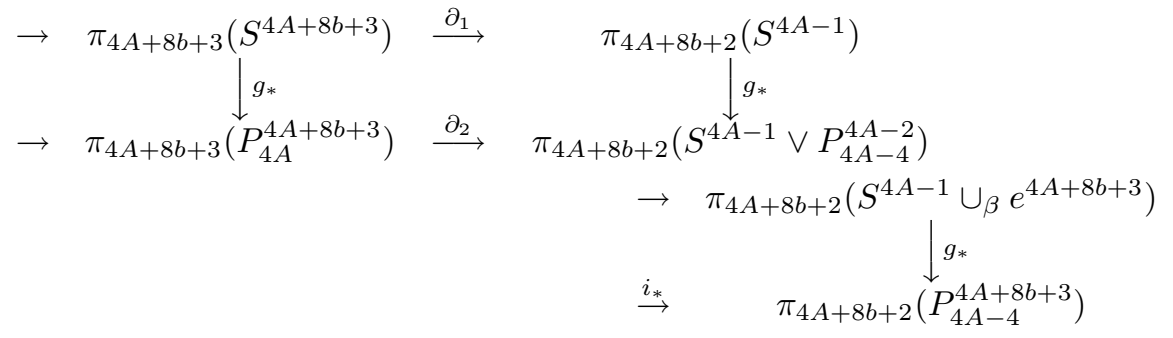

whose rows are exact sequences. Then $\partial_{1}(\iota)=\beta, \partial_{2}\left(g_{*}(\iota)\right)=x_{3}$. This means $i_{*} x_{3}=0$ in $\pi_{*}\left(P_{4 A-4}^{4 A+8 b+3}\right)$ and (i) follows.

Consider (ii). If $\nu(4 A+8 b+4) \leq 4 b+2$, then (ii) follows from (i) by the map $\rho: P_{4 A-4}^{4 A+8 b+3} \rightarrow L_{4 A-4}^{4 A+8 b+3}$. Suppose $\nu(4 A+8 b+4)=4 b+3$. Let $\lambda=\lambda_{8 b+7}$ and $D L_{4 A-4}^{4 A+8 b+7}=\Sigma L_{n}^{n+8 b+7}$. Then $\nu(n)=4 b+3$. Thus $\frac{n}{4} \lambda$ is stably trivial over $L^{8 b+3}$, and $\frac{n}{2} \bar{\lambda}=p^{*}(2 \eta)$, where $\eta$ is a stable vector bundle over $S^{8 b+4}$ corresponding to a generator of $\widehat{K O}\left(S^{8 b+4}\right) \otimes \mathbf{Z}_{(2)}$. Then the proof follows just as (i).

Lemma 6.3. (i) Let $\nu(4 A+8 b)=2$ and $b \geq 2$. The composite

$$
x_{5}: S^{4 A+8 b-2} \stackrel{4 \beta_{8 b-1}}{\longrightarrow} S^{4 A-1} \rightarrow L_{4 A-4}^{4 A-1}
$$

is null-homotopic in $L_{4 A-4}^{4 A+6}$.

(ii) If $3 \leq \nu(4 A+8) \leq 4$, then the composite $x_{5}^{\prime \prime}: S^{4 A+6} \stackrel{2 \beta_{7}}{\longrightarrow} S^{4 A-1} \rightarrow L_{4 A-4}^{4 A-1}$ is null-homotopic in $L_{4 A-4}^{4 A+7}$.

Proof. Consider (i). Let $\partial, \partial^{\prime}$ be respectively the boundaries from $\pi_{*}\left(P_{4 A+3}^{4 A+6}\right)$, $\pi_{*}\left(L_{4 A+3}^{4 A+6}\right)$ to $\pi_{*-1}\left(P_{4 A-4}^{4 A+2}\right), \pi_{*-1}\left(L_{4 A-4}^{4 A+2}\right)$. Let $i$ be a suitable inclusion. Let $x_{5}^{\prime}$ be the composite $S^{4 A+8 b-2} \stackrel{\beta_{8 b-1}}{\longrightarrow} S^{4 A-1} \rightarrow P_{4 A-4}^{4 A-1}$. Then $x_{5}=2 \rho\left(x_{5}^{\prime}\right)$. Lemma 4.2 (ii) implies $x_{5}^{\prime}$ is null in $J_{4 A+8 b-2}\left(P_{4 A-4}^{4 A+6}\right)$ but not null in $J_{4 A+8 b-2}\left(P_{4 A-4}^{4 A+5}\right)$. So each element $x \in J_{4 A+8 b-1}\left(P_{4 A+3}^{4 A+6}\right)$ that is from the nontrivial element

$$
w_{1} \in E_{2}=H_{4 A+6}\left(P_{4 A+3}^{4 A+6} ; J_{8(b-1)+1}\right) \approx \mathbf{Z}_{2}
$$

in AHSS will satisfy $\partial(x)=i_{*}\left(x_{5}^{\prime}\right)$ when put into $J_{*}\left(P_{4 A-4}^{4 A+2}\right)$. By Lemma 4.3, it is easy to see that $w_{1}: S^{4 A+8 b-1} \stackrel{\beta_{8(b-1)+1}}{\longrightarrow} S^{4 A+6}$ coextends to a map $w_{2}$ : $S^{4 A+8 b-1} \rightarrow P_{4 A+3}^{4 A+6}$ of Adams filtration $4(b-1)$. Thus $A\left(\partial\left(w_{2}\right)\right) \geq 4 b-3$, so $\partial^{\prime}\left(2 \rho_{*}\left(w_{2}\right)\right)$ is of Adams filtration $\geq 4 b-2$, and $\partial^{\prime}\left(2 \rho_{*}\left(w_{2}\right)\right)=i_{*}\left(x_{5}\right)$ when put into $J_{*}\left(L_{4 A-4}^{4 A+2}\right)$ because $\partial\left(w_{2}\right)=i_{*}\left(x_{5}^{\prime}\right)$ when put into $J_{*}\left(P_{4 A-4}^{4 A+2}\right)$ and because $x_{5}=2 \rho\left(x_{5}^{\prime}\right)$. In ASS the chart for $E_{2}^{s, t}\left(L_{4 A-4}^{4 A+2}\right)$ with $s \geq 4 b-2$ and $4 A+8 b-2 \leq t-s \leq 4 A+8 b-1$ is given in the below

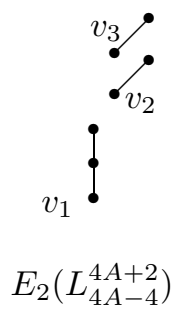


where $v_{1}$ is in the position $(t-s, s)=(4 A+8 b-2,4 b-2)$ surviving to an element in $J_{*}\left(L_{4 A-4}^{4 A+2}\right)$ of Adams filtration $4 b-2$. Since $A\left(i_{*}\left(x_{5}\right)\right)=4 b-1, \partial^{\prime}\left(2 \rho_{*}\left(w_{2}\right)\right)=i_{*}\left(x_{5}\right)$ because $\eta\left(x_{5}\right)=0$, while $\eta$ is injective on classes to which $v_{2}$ and $v_{3}$ converge.

Consider (ii). As in Lemma 6.2, if $\nu(4 A+8)=3$ the composite

$$
S^{4 A+6} \stackrel{\beta_{7}}{\longrightarrow} S^{4 A-1} \rightarrow P_{4 A-4}^{4 A+7}
$$

is null-homotopic and (ii) follows by the map $\rho$. The case $\nu(4 A+8)=4$ can be proved in the same way by noting that $((4 A+8) / 2) \lambda_{11}$ is stably trivial over $L^{7}$.

Lemma 6.4. (i) There is a map $i_{0}: S^{4 A-1} \cup_{\eta} e^{4 A+1} \rightarrow P_{4 A-2}^{4 A+1}$ such that $g_{*}$ on $H_{*}\left(-; \mathbf{Z}_{2}\right)$ is injective.

(ii) $S^{4 A+8 b+2} \stackrel{\beta_{8 b+1}}{\longrightarrow} S^{4 A+1}$ coextends to a map $w: S^{4 A+8 b+2} \rightarrow S^{4 A-1} \cup_{\eta} e^{4 A+1}$ such that $A(w) \geq 4 b$, and $\left(\rho i_{0}\right)_{*}(w)=z$ in $\pi_{*}\left(L_{4 A-2}^{4 A+1}\right)$, where $z$ is the composite

$$
S^{4 A+8 b+2} \stackrel{\beta_{8 b+3}}{\longrightarrow} S^{4 A-1} \rightarrow S^{4 A-1} \vee S^{4 A-2}=L_{4 A-2}^{4 A-1} \rightarrow L_{4 A-2}^{4 A+1} .
$$

(iii) If $\nu(4 A+8 b+4)=2$, then $i_{0} w$ is null-homotopic in $\pi_{*}\left(P_{4 A-2}^{4 A+8}\right)$ (hence $z$ is null-homotopic in $\left.\pi_{*}\left(L_{4 A-2}^{4 A+8}\right)\right)$.

Proof. Consider (i). Let $D P_{4 A-2}^{4 A+1}=\Sigma P_{2 B}^{2 B+3}=\Sigma T(2 B \xi)$, where $\xi$ is the real Hopf bundle over $P^{3}$. There is a stable vector bundle $\eta^{\prime}$ over $S^{2}$ corresponding to the generator of $\widetilde{K O}\left(S^{2}\right)$ with $p^{*}\left(\eta^{\prime}\right)=2 B \bar{\xi}$. So we have a map $f_{1}: P_{2 B}^{2 B+3} \rightarrow T\left(\eta^{\prime}\right)=$ $S^{2 B} \cup_{\eta} e^{2 B+2}$ inducing a monomorphism on $H^{*}\left(-; \mathbf{Z}_{2}\right)$, where $\eta=\beta_{1}$. Thus (i) follows by duality.

The first half of (ii) is from [4, Lemma 6$]$ by applying the map $i_{0}$. Consider the second half. In the ASS, the chart for $E_{3}^{s, t}\left(L_{4 A-2}^{4 A+1}\right)$ with $s \geq 4 b$ and $t-s=4 A+8 b+2$, is as follows.

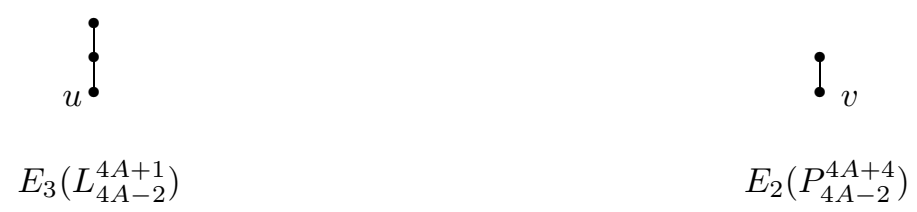

where $u$ is in the position $(t-s, s)=(4 A+8 b+2,4 b)$ and $2 u$ corresponds to $z$ in homotopy. Since $\left(\rho i_{0}\right)^{*}$ induces zero morphism on $H^{*}\left(-; \mathbf{Z}_{2}\right)$, we have $A\left(\rho\left(i_{0}\right)(w)\right) \geq 4 b+1$. Putting $\rho i_{0}(w)$ into $J_{*}(-)$, we see $A\left(\rho i_{0}(w)\right)=4 b+1$. Thus $\left(\rho i_{0}\right)_{*}(w)$ must be $z$.

Consider (iii). Lemma 4.2 (iv) implies that $i_{0} w$ is null in $J_{4 A+8 b+2}\left(P_{4 A-2}^{4 A+8}\right)$ but not null in $J_{4 A+8 b+2}\left(P_{4 A-2}^{4 A+7}\right)$. As before, each coextension $x: S^{4 A+8 b+3} \rightarrow P_{4 A+5}^{4 A+8}$ of $4 \beta_{8(b-1)+3}: S^{4 A+8 b+3} \rightarrow S^{4 A+8}$ will satisfy $\partial(x)=i_{0} w$ in $J_{*}\left(P_{4 A-2}^{4 A+4}\right)$. By Lemma 4.3 , there exists a coextension $x: S^{4 A+8 b+3} \rightarrow P_{4 A+5}^{4 A+8}$ of $4 \beta_{8(b-1)+3}$ with $A(x) \geq$ $4(b-1)+3$, hence $A(\partial(x)) \geq 4 b$. Then $\partial(x)=i_{0} w$ follows from the chart $E_{2}^{s, t}\left(P_{4 A-2}^{4 A+4}\right)$ with $s \geq 4 b$ and $t-s=4 A+8 b+2$ as given above. Here $v$ is in the position $(t-s, s)=(4 A+8 b+2,4 b)$, and corresponds to $i_{0} w$ when put into $P_{4 A-2}^{4 A+4}$, because $A\left(i_{0} w\right)=4 b$.

The case $\nu(4 A+8 b+4) \geq 3$ in Lemma 6.4 is handled in the next lemma. 
Lemma 6.5. Let $i_{0}$ and $w, z$ be as in Lemma 6.4.

(i) If $3 \leq \nu(4 A+8 b+4) \leq 4 b+1$, then the composite

$$
x_{6}: S^{4 A+8 b+2} \stackrel{i_{0} w}{\longrightarrow} P_{4 A-2}^{4 A+1} \rightarrow P_{4 A-2}^{4 A+8 b+3}
$$

is null-homotopic.

(ii) If $3 \leq \nu(4 A+8 b+4) \leq 4 b+2$, then $z$ is null in $\pi_{*}\left(L_{4 A-2}^{4 A+8 b+3}\right)$.

Proof. Part (i) is implied by Lemma 6.4 (ii) and Lemma 5.8. Consider (ii). Note that $z=\rho x_{6}$. If $3 \leq \nu(4 A+8 b+4) \leq 4 b+1$, then $z$ is null-homotopic in $\pi_{*}\left(L_{4 A-2}^{4 A+8 b+3}\right)$ by (i). If $\nu(4 A+8 b+4)=4 b+2$, let $\lambda=\lambda_{8 b+5}$, then $\frac{n}{2} \lambda$ is stably trivial over $L^{8 b+3}$, and (ii) follows from an argument as in the proof of Lemma 6.2.

\section{REFERENCES}

[1] J. F. Adams, Vector fields on spheres, Ann. of Math., Vol.75 No.3 (1962) 603-632. MR 25:2614

[2] J. F. Adams, On the non-existence of elements of Hopf invariant one, Ann. of Math. 72 (1960), 20-103. MR 25:4530

[3] M. F. Atiyah, Thom complexes, Proc. London Math. Soc. 11 (1961), 291-310. MR 24:A1727

[4] D. M. Davis and M. E. Mahowald, Classification of the stable homotopy types of stunted real projective spaces, Pacific J. Math., Vol.125 No.2 (1986) 335-345. MR 88a:55008

[5] D. M. Davis, Generalized homology and the generalized vector field problem, Quart. J. Math. Oxford, 25 (1974), 161-193. MR 50:8524

[6] D. M. Davis and M. E. Mahowald, Homotopy groups of some mapping telescopes, Ann. of Math. Stud., no.113, Princeton Univ. Press (1987), 126-151. MR 89a:55013

[7] S. Feder, S. Gitler, and M. Mahowald, On the stable homotopy types of stunted projective spaces, Bol. Soc. Mat. Mex., 22 (1977), 1-5. MR 81b:55017

[8] K. Fujii, T. Kobayashi and M. Sugawara, Stable homotopy types of stunted lens spaces, Mem. Fac. Sci. Kochi Univ. (Math.), 3(1982), 21-27. MR 83h:55008

[9] Jesus Gonzalez, Classification of the stable homotopy types of stunted lens spaces mod p, to appear.

[10] D. Husemoller, Fibre bundles, Second edition, Springer-Verlag, New York, Heidelberg, Berlin, 1975. MR 51:6805

[11] T. Kobayashi and M. Sugawara, On stable homotopy types of stunted lens spaces, Hiroshima Math. J. 1 (1971), 287-304. MR 47:1062

[12] T. Kobayashi and M. Sugawara, Note on KO-rings of lens spaces mod $2^{r}$, Hiroshima Math. J. 8 (1978), 85-90. MR 80e:55033

[13] S. Kono, Stable homotopy types of stunted lens spaces mod 4, Osaka J. Math. 29 (1992), 697-717. MR 93i:55009

[14] S. Kono and A. Tamamura, J-groups of suspensions of stunted lens spaces mod 4, Osaka J. Math., 26 (1989), 319-345. MR 90m:55014

[15] K. Y. Lam, KO-equivalences and existence of nonsingular bilinear maps, Pacific J. Math., 82(1979), 145-153. MR 81a:55022

[16] Mark Mahowald, The metastable homotopy of $S^{n}$, Mem. Amer. Math. Soc., 72 (1967). MR 38:5216

[17] Mark Mahowald, The image of $J$ in the EHP sequence, Annals of Math. 116(1982), 65-112. MR 83i:55019

[18] D. Ravenel, Complex cobordism and stable homotopy groups of spheres, Academic Press, New York (1986). MR 87j:55003

[19] R. M. Switzer, Algebraic Topology-Homotopy and Homology, Springer-Verlag, Berlin, Heidelberg (1978). MR 52:6695

[20] A. Tamamura and S. Kono, On the KO-cohomologies of the stunted lens spaces, Math. J. Okayama Univ. 29 (1987), 233-244. MR 89g:55007

[21] G. W. Whitehead, Elements of homotopy theory, Springer-Verlag, New York, Heidelberg, Berlin (1978). MR 80b:55001

Department of Mathematics, Lehigh University, Bethlehem, Pennsylvania 18015 